\title{
Cooperative Load Balancing in Hybrid Visible Light Communications and WiFi
}

\author{
Xuan Li, Rong Zhang, Member, IEEE, and Lajos Hanzo
}

\begin{abstract}
As a complementary extension of established Radio Frequency (RF) Wireless Local Area Networks (WLANs), Visible Light Communication (VLC) using commercially available Light-Emitting Diode (LED) transmitters offers a huge data rate potential in this license-free spectral domain, whilst simultaneously satisfying energy-efficient illumination demands. Various VLC cell formations, ranging from a regular cell-layout associated with different Frequency Reuse (FR) patterns to merged cells by employing advanced transmission scheme are investigated. Furthermore, a hybrid Down-Link (DL) offering full RF-coverage by a WLAN and additionally supported by the abundant spectral resources of a VLC network is studied. Cooperative Load Balancing (LB) achieving Proportional Fairness (PF) is implemented by using both centralized and distributed resource-allocation algorithms. The performance of this hybrid RF/VLC system is analysed both in terms of its throughput and fairness in diverse cell formation scenarios. Our simulation results demonstrate that, the VLC system advocated is capable of providing a high Area Spectral Efficiency (ASE) and our hybrid RF/VLC system achieves the highest throughput and the highest grade of fairness in most of the scenarios considered.
\end{abstract}

Index Terms-Cell formation, cooperative load balancing, heterogeneous networks, visible light communication.

\section{INTRODUCTION}

W ITH the promise of gaining access to a huge unlicensed bandwidth, which is available in the optical domain of the electromagnetic spectrum, the research of Optical Wireless (OW) communications intensified during the past decade or so [1]. Apart from the substantial amount of research on the infrared region of the optical spectrum [2], [3], as a benefit of the rapid development of solid-state lighting, high data rate Visible Light Communication (VLC) combined with advanced illumination has become a reality in indoor scenarios [4]-[6]. Specifically, the Light-Emitting Diodes (LEDs) exhibit a high energy efficiency and additionally they are capable of exploiting a vast unregulated spectrum. Extensive investigations have

Manuscript received November 15, 2013; revised May 1, 2014, October 1, 2014, and January 14, 2015; accepted February 24, 2015. The financial support of the RC-UK under the auspices of the UK-India ATC in Wireless Communications, of the Chinese Scholarship Council as well as of the European Research Council's Advanced Fellow Grant is gratefully acknowledged. The associate editor coordinating the review of this paper and approving it for publication was H. Haas.

The authors are with the Communications, Signal Processing and Control, School of ECS, University of Southampton, Southampton SO17 1BJ, U.K. (e-mail: x126g12@ecs.soton.ac.uk; rz@ecs.soton.ac.uk; 1h@ecs.soton.ac.uk).

Color versions of one or more of the figures in this paper are available online at http://ieeexplore.ieee.org.

Digital Object Identifier 10.1109/TCOMM.2015.2409172 been dedicated to the physical layer of VLC [7]-[11], as also indicated by the IEEE 802.15.7 standard ratified for shortrange visible light wireless communication [12]. As far as the network level of our VLC system is concerned, stand-alone VLC networks may exhibit some potential drawbacks, such as for example: 1) VLC networks perform poorly in nonline-of-sight scenarios owing to the predominantly Line-OfSight (LOS) propagation of light; 2) In VLC networks, each optical Access Point (AP) illuminates only a small confined cell compared to cellular RF networks; 3) VLC networks fail to provide convenient Up-Link (UL) coverage at the current-stateof-the-art. To overcome the above drawbacks, it is necessary to develop cooperative Heterogeneous Networks (HetNets), which additionally rely on Radio Frequency (RF) techniques as a complementary extension. As a result, the widely used Wireless Fidelity (WiFi) network may be invoked as a cooperative partner of the VLC networks in indoor scenarios. As mentioned above, the proposed hybrid VLC/WiFi system is capable of providing high-data-rate connections as well as a seamless reliable coverage [1]. Compared to the traditional WiFi-only and VLC-only systems, the integration of VLC and WiFi is expected to significantly improve the aggregate throughput, which has been shown both by analytical and simulation results in [13] and the independent efforts disseminated in [14] led to similar conclusions. This motivates our research of the hybrid VLC/WiFi system by investigating fair and efficient cooperative Load Balancing (LB), where a salient problem is the appropriate formation of VLC cells.

There is a paucity of studies on the formulation of VLC cells, although recently some valuable studies were disseminated in the context of a stand-alone VLC-only system. In particular, the authors of [15] discussed Fractional Frequency Reuse (FFR) for VLC cells and subsequently a joint transmission regime was derived in [16] for VLC cells. ${ }^{1}$ On the other hand, LB constitutes one of the fundamental problems, which in fact affects all cooperative multi-rate HetNets. Broadly speaking, this problem can be formulated as the constrained optimization of a carefully selected utility function [17]. Substantial related work has been undertaken based on this problem in RF networks [18]-[25]. Specifically, the authors of [18] investigated diverse technical approaches to the LB problems of HetNes and provided valuable design guidelines for OFDMA-based cellular systems. Moreover, the authors of [19]-[21] proposed centralized solutions, which rely on a centralized resource manager,

\footnotetext{
${ }^{1}$ Our combined transmission proposed in this article is reminiscent of the joint transmission introduced in [16], which was published after our submission. Hence these two works are independently developed.
} 
while the authors of [22]-[25] addressed the LB problems of cellular networks with the aid of distributed algorithms. More particularly, Burchardt et al. [22] introduced a fuzzy logic based system, while Heliot et al. [23] proposed the employment of the Newton-Raphson-based method. However, both of them considered a homogeneous single-network scenario, rather than a VLC/RF HetNet scenario. As a further advance, in [24], [25] the LB problem of a RF-based HetNet was solved by using the dual decomposition approach and provided a near-optimal solution at a low complexity. However, the LB problem of VLC-based HetNets has remained to a large extent hitherto unexplored [26], especially when combined with various VLC cell formations.

Against the above-mentioned background, in this paper, we investigate the LB problem between several VLC APs and a WiFi AP relying on the users' Proportional Fairness (PF) as a measure, when jointly considering various VLC cell formations, ranging from traditional cellular design to merged cells.

The rest of this paper is organized as follows. Our hybrid system model and the various cell formations considered are presented in Section II.

Our methodology for solving this LB problem, including both the centralized as well as its decomposed/distributed counterpart are described and evaluated in Section III and Section IV, respectively. Finally, Section V offers our conclusions.

Notation: Bold variables are used to denote vectors, matrices or sets, while regular Roman characters are used for general parameters.

\section{SYSTEM MODEL}

A hybrid VLC/WiFi Down-Link (DL) system model is considered, where the IEEE 802.11 Wireless Local Area Network (WLAN) is complemented by an optical network. The hybrid network has a set of VLC APs as well as a WiFi AP, but this scenario may be readily extended to other AP configurations. More explicitly, each VLC AP relies on an LED lamp constructed from several LEDs. Let us first discuss a range of VLC cell formations in this section before investigating our LB problems.

\section{A. Link Characteristic}

For a given transmitted optical power $P_{t}$ of each VLC AP, the average optical power $P_{r}$ received by a photodetector is the sum of the power received from all the corresponding transmitting VLC APs within its vicinity, which is hosted in the set $\boldsymbol{S}$ and it is given as ${ }^{2}$

$$
P_{r}=\sum_{i \in S} P_{r, i}=\sum_{i \in S} H_{i}(0) P_{t}
$$

\footnotetext{
${ }^{2}$ Since our major concern is that of investigating various VLC cell formations and finding an efficient LB solution for this hybrid system, some of the practical VLC channel characteristics have been simplified. The optical channel of (2) may be widely adopted, when considering a Lambertian source in indoor optical wireless scenarios. Our algorithm is a generic one, which may be readily adapted to other types of optical channels.
}

TABLE I

VLC PARAMETERS

\begin{tabular}{l|r}
\hline \hline Transmitted optical power per LED lamp $\left(P_{t}\right)$ & $20[\mathrm{~W}]$ \\
Semi-angle at half power $\left(\phi_{1 / 2}\right)$ & $60^{\circ}$ \\
Modulation bandwidth $(B)[4],[5],[9],[11]$ & $20[\mathrm{MHz}]$ \\
Physical area of a PD $\left(D_{\mathrm{PA}}\right)$ & $1.0\left[\mathrm{~cm}^{2}\right]$ \\
Gain of an optical filter $\left(T_{s}(\psi)\right)$ & 1.0 \\
Refractive index of a lens at a PD $(n)$ & 1.5 \\
O/E conversion efficiency $(\gamma)$ & $0.53[\mathrm{~A} / \mathrm{W}]$ \\
Half of the receiver's FOV $\left(\psi_{\mathrm{F}}\right)$ & $60^{\circ} / 65^{\circ}$ \\
BER threshold & $10^{-5}$ \\
Roll-off factor $(\rho)$ & 1 \\
Normalized WiFi capacity for DL $\left(p_{\mathrm{DL}}\right)$ & 0.8 \\
Supply and demand gap $\left(\delta_{\text {target }}\right)$ & 1 \\
\hline \hline
\end{tabular}

According to [3], [27], by dropping the index $i$ without loss of generality, the optical channel's total Direct Current (DC) attenuation from each VLC AP to the receiver is given by

$$
H(0)= \begin{cases}\frac{(m+1) D_{\mathrm{PA}}}{2 \pi r^{2}} \cos ^{m}(\phi) T_{s}(\psi) g(\psi) \cos (\psi), & \psi \leq \psi_{\mathrm{F}}, \\ 0, & \psi>\psi_{\mathrm{F}},\end{cases}
$$

where the Lambert index $m$ depends on the semi-angle $\phi_{1 / 2}$ at half-illuminance of the source, which is given by $m=$ $-1 / \log _{2}\left(\cos \phi_{1 / 2}\right) \cdot D_{\mathrm{PA}}$ is the detector's physical area for a Photo-Diode (PD), $r$ is the distance between the VLC transmitter and the receiver, $\phi$ is the angle of irradiance, $\psi$ is the angle of incidence and $\psi_{\mathrm{F}}$ represents half of the receiver's Field-OfView (FOV). In a direct LOS path, the irradiant angle equals to the incident angle. Still referring to (2), $T_{s}(\psi)$ and $g(\psi)$ denote the gain of the optical filter and of the optical concentrator employed, respectively, while $g(\psi)$ can be written as [3]

$$
g(\psi)= \begin{cases}\frac{n^{2}}{\sin ^{2} \psi_{\mathrm{F}}}, & 0 \leq \psi \leq \psi_{\mathrm{F}} \\ 0, & \psi>\psi_{\mathrm{F}}\end{cases}
$$

where $n$ is the refractive index of a lens at a PD. Our parameter values are summarized in Table I.

According to [6], the average received power including all reflections may be negligible compared with the direct received average power of the LOS path. Therefore we may ignore the reflected optical power for simplicity and consider only the LOS-power as the desired received power. As a result, when the incoming optical radiation having an average power $P_{r}$ impinging on a photodetector, the electronic current generated by the photodetector is given by

$$
\left\langle i_{\mathrm{PD}}\right\rangle=\gamma \cdot P_{r},
$$

where $\gamma[\mathrm{A} / \mathrm{W}]$ denotes the photodetector's responsivity. Let us now define the Signal to Interference plus Noise Ratio (SINR) as the aggregate electronic power received from signal set $\boldsymbol{S}_{S} \subseteq \boldsymbol{S}$ over the noise power in a bandwidth of $B$ [MHz] [5] plus the sum of the electronic power received from other optical sources in interference set $\boldsymbol{S}_{I}$, which is the complementary set of $\boldsymbol{S}_{S}$. Since the corresponding electronic power is proportional 
to the square of the amplitude of the electronic current, we can write the SINR as

$$
\xi=\frac{\gamma^{2} \sum_{i \in \boldsymbol{S}_{S}} P_{r, i}^{2}}{N_{0} B+\gamma^{2} \sum_{i \in S_{I}} P_{r, i}^{2}}
$$

where $N_{0}\left[\mathrm{~A}^{2} / \mathrm{Hz}\right]$ is the noise power spectral density dominated by the shot noise $N_{\text {shot }}$ [6], given as $N_{0} \cong N_{\text {shot }}=$ $q I_{a}\left(P_{r}\right) \sim 10^{-22}$, where $q$ denotes the electron charge and $I_{a}\left(P_{r}\right)$ is the photo-current at the receiver [5], which relies on the received power. The expression in (5) is in its common form and it will be different for each of the VLC cell formations, which are discussed next.

\section{B. Regular Cell Formation}

1) Unity Frequency Reuse: The most straightforward way of constructing a VLC cell is to simply assume that each VLC AP illuminates an individual cell, which corresponds to adopting Unity Frequency Reuse (UFR) across all cells. Fig. 1(a) shows the UFR design, where each single VLC AP illuminates an individual cell and the same frequency $f$ is reused across all cells. The shaded areas represent the Inter-Cell Interference (ICI) imposed by the LOS ray conveying different information and arriving from the neighbouring cells at the cell edge. For the triangular point shown in Fig. 1(a), the SINR is given by

$$
\xi_{\mathrm{UFR}}=\frac{\gamma^{2} P_{r, \mathrm{~A}}^{2}}{N_{0} B+\gamma^{2}\left(P_{r, \mathrm{~B}}^{2}+P_{r, \mathrm{C}}^{2}+P_{r, \mathrm{D}}^{2}\right)},
$$

which shows that ICI arrives from all the other three neighbouring cells in this scenario. If the FOV was sufficiently narrow, the ICI may be mitigated since the user can only receive data from a single VLC AP. However, this would potentially lead to isolated "coverage islands" and "coverage holes," which consequently may result into frequent horizontal handovers and outage event, when the DL user is walking between VLC APs, since the user will experience dramatic performance degradation in the area without LOS coverage.

2) Non-Unity Frequency Reuse: Following the traditional cellular design principle, non-unity Frequency Reuse (FR) patterns may be employed for reducing the ICI, while each VLC AP still functions as an individual cell. Since the first-tier neighbouring cells contribute most of the ICI, while the secondtier cells generally have a negligible influence, an FR factor of two may be used. For the triangular point shown in Fig. 1(b), the SINR is given by

$$
\xi_{\mathrm{FR}-2}=\frac{\gamma^{2} P_{r, \mathrm{~A}}^{2}}{N_{0} B / 2+\gamma^{2} P_{r, \mathrm{D}}^{2}} .
$$

Hence, the ICI emanating from the neighbouring cells $B$ and $C$ can be removed. Although this is an appealingly simple solution, when using an FR factor larger than one, the system has to obey the classic trade-off between reduced Bandwidth

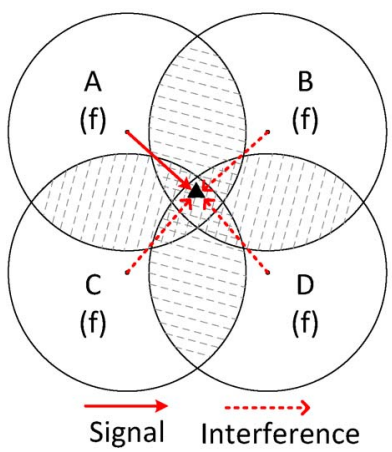

(a)

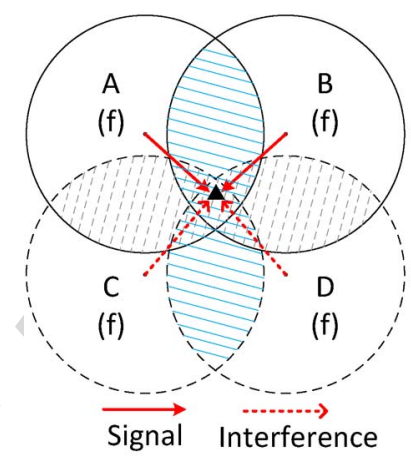

(c)

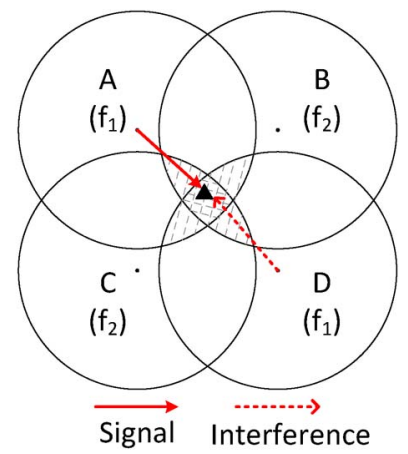

(b)

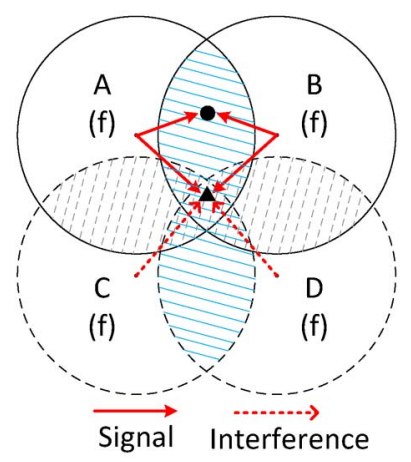

(d)
Fig. 1. Different cell formations. (a) A regular cell formation, (b) has a FR factor of two, (c) represents two merged 2-AP cells with CT, and (d) shows two merged 2-AP cells using VT. The triangle and circle denote certain points of reception. The shaded areas covered with dotted lines represent the ICI imposed by the LOS ray of neighbouring cells at the cell edge. The shaded areas covered by solid lines represent the overlapping areas within the merged 2-AP cells. In (a), (c), and (d), the entire frequency band $f$ is used by each small cell, while in (b) orthogonal frequencies $f_{1}$ and $f_{2}$ are employed by neighbouring cells, where we have $f_{1}=f_{2}=f / 2$.

Efficiency (BE) and improved cell-edge SINR. In fact, supporting mobility is the most grave problem associated with nonunity FR during VLC cell formation, since switching between frequencies every few meters during the user's movement degrades the user experience. This is also the reason for not considering FFR [15], which exhibits a more elaborate frequency planning and triggers even more frequent handovers.

\section{Merged Cell Formation}

To reduce the size of the ICI-infested areas, whilst improving the mobility, several neighbouring cells can be merged into a large multi-AP cell, where advanced transmission techniques may be employed in their overlapping areas. In the following, we use UFR across multi-AP cells for simplicity, although nonunity FR might be also used.

1) Combined Transmission: In this arrangement, each individual VLC AP of a multi-AP cell conveys the same information on the same visible carrier frequency in their overlapping areas. In Fig. 1(c), $A$ and $B$ are merged into a 2-AP cell and transmit identical signals in their overlapping area as a single source, which we refer to as Combined Transmission (CT). Thus the potential ICI is beneficially turned into useful signals which may be combined and the original cell edges of Fig. 1(a) 
become the cell centers of Fig. 1(c). For the triangular point shown in Fig. 1(c), the SINR is given by

$$
\xi_{\mathrm{CT}-2}=\frac{\gamma^{2}\left(P_{r, \mathrm{~A}}^{2}+P_{r, \mathrm{~B}}^{2}\right)}{N_{0} B+\gamma^{2}\left(P_{r, \mathrm{C}}^{2}+P_{r, \mathrm{D}}^{2}\right)} .
$$

Although the SINR may be enhanced, CT results in a reduced $\mathrm{BE}$, since only a single user is served at a time by several APs in the overlapping area within a merged cell.

2) Vectored Transmission: To eliminate the BE-reduction imposed by CT, Zero-Forcing (ZF)-based Vectored Transmission (VT) techniques can be employed for serving multiple users at the same time in the overlapping area. The underlying principle of ZF-based VT is to totally eliminate the ICI at the multiple transmitters, so that the multiple users receive mutually interference-free signals.

To elaborate a little further, let $n_{\alpha}$ denote the number of APs in a merged $n_{\alpha}$-AP cell and a vector of $U$ users are served simultaneously within the overlapping area. Let further $\boldsymbol{x}_{\boldsymbol{t}} \in \mathbb{R}^{U \times 1}$ and $\boldsymbol{y}_{\boldsymbol{r}} \in \mathbb{R}^{U \times 1}$ denote the vectors of transmitted and received signals, respectively. By using VT, we have

$$
\boldsymbol{y}_{\boldsymbol{r}}=\gamma P_{t} \boldsymbol{H} \boldsymbol{G} \boldsymbol{\Omega} \boldsymbol{x}_{\boldsymbol{t}}+\boldsymbol{n},
$$

where $\boldsymbol{n}$ includes both the noise and the ICI received from the neighbouring merged cells, while the channel-matrix $\boldsymbol{H} \in$ $\mathbb{R}^{U \times n_{\alpha}}$ hosts the DC attenuations between $U$ users and $n_{\alpha}$ APs. Furthermore, the matrix $\boldsymbol{G}=\boldsymbol{H}^{H}\left(\boldsymbol{H} \boldsymbol{H}^{H}\right)^{-1}$ obeys the ZF criterion, which hence results in a beneficial ICI-free identity matrix for $\boldsymbol{H} \boldsymbol{G}=\boldsymbol{I}_{U}$. Finally, the matrix $\boldsymbol{\Omega}$ is introduced to enforce the per-AP power constraints. According to [28], we have

$$
\boldsymbol{\Omega}=\omega \boldsymbol{I}_{U}, \quad \omega=\min _{\alpha=1,2, \ldots, n_{\alpha}} \sqrt{\frac{1}{\|\boldsymbol{G}(\alpha,:)\|_{F}^{2}}},
$$

where $\boldsymbol{G}(\alpha,:)$ is the $\alpha$ th row of $\boldsymbol{G}$. As a result, the SINR of a particular user may be written as ${ }^{3}$

$$
\xi_{\mathrm{JT}}=\frac{\gamma^{2} P_{t}^{2} \omega^{2}}{N_{0} B+\gamma^{2} \sum_{i \in \boldsymbol{S}_{I}} P_{r, i}^{2}} .
$$

In general, to facilitate VT from $n_{\alpha}$ APs to $U$ users, both the $\left(U \times n_{\alpha}\right)$-element DC attenuation matrix and the users' data have to be shared amongst the $n_{\alpha}$ APs [29]. For VLC channels, the requirements may be readily satisfied, since the VLC users are predominantly stationary. This is similar to the successful employment of ZF-based Transmit Pre-Coding (TPC) techniques, which is referred to as VT in the stateof-the-art Digital Subscriber Line (DSL) based G.fast system (Recommendation ITU-T G.9701) invoked for coping with the Far End Cross Talk (FEXT) between twisted pairs.

\footnotetext{
${ }^{3}$ Since the interference power received by the merged cell under consideration is influenced by the ZF-based VT within its neighbouring merged cells, for simplicity, we assume that the interference imposed is always equal to its maximum value, which characterizes the worst-case situation in our VT cell formation scenario.
}

As an example, in Fig. 1(d), $A$ and $B$ form a VT-aided 2-AP cell and two users are served simultaneously in the originally ICI-infested areas. For the paired points shown in Fig. 1(d), the SINRs are given by

$$
\xi_{\mathrm{JT}-2}^{\bullet}=\frac{\gamma^{2} P_{t}^{2} \omega^{2}}{N_{0} B} ; \quad \xi_{\mathrm{JT}-2}^{\boldsymbol{\Delta}}=\frac{\gamma^{2} P_{t}^{2} \omega^{2}}{N_{0} B+\gamma^{2}\left(P_{r, \mathrm{C}}^{2}+P_{r, \mathrm{D}}^{2}\right)} .
$$

\section{Area Spectral Efficiency}

To gain further quantitative insights, Fig. 2 illustrates both the classic BE surface and the Mean Bandwidth Efficiency (MBE) of different VLC cell formations. The BE is calculated as

$$
\begin{aligned}
\eta_{\mathrm{UFR}} & =\log _{2}\left(1+\xi_{\mathrm{UFR}}\right) ; \quad \eta_{\mathrm{FR}-2}=\frac{1}{2} \log _{2}\left(1+\xi_{\mathrm{FR}-2}\right) ; \\
\eta_{\mathrm{CT}} & =\hbar \log _{2}\left(1+\xi_{\mathrm{CT}-2}\right) ; \quad \eta_{\mathrm{JT}}=\log _{2}\left(1+\xi_{\mathrm{JT}}\right) ;
\end{aligned}
$$

where $\hbar$ accounts for the loss of resource under utilisation of CT, which is 0.9122 and 0.8737 when the FOV is $120^{\circ}$ and $125^{\circ}$ under our simulation setup, respectively. ${ }^{4}$ In our simulations, a $15 \mathrm{~m} \times 15 \mathrm{~m} \times 3 \mathrm{~m}$ room model is considered, including $4 \times 4$ uniformly distributed optical APs at a height of $2.5 \mathrm{~m}$. The parameters of the VLC APs are summarized in Table I. Compared to UFR as shown in Fig. 2(a), a FR of two sufficiently reduces the ICI-contaminated areas, but results into a significantly reduced $\mathrm{BE}$ as shown in Fig. 2(b). By contrast, all of our proposed merged cell formations shown in Fig. 2(c)-(e) improve the MBE, as suggested by Fig. 2(f). More explicitly, when a 2-AP cell is created, the MBE of VT is only marginally better than that of CT. However, when forming all VLC APs as a single cell, a substantial MBE improvement can be achieved with the aid of VT, since the resultant system becomes reminiscent of a large-scale Multi-Input Multi-Output (MIMO) system.

\section{Methodology}

Let us now determine the optimum LB for a set of users in this hybrid VLC/WiFi system by taking into account various VLC cell formations. We would like to introduce some notations first. Let $\boldsymbol{C}$ be the set of the single-AP or multi-AP VLC cells, where $|\boldsymbol{C}|=C$ is the number of cells. In this section, we illustrate our methodology in the UFR scenario, which can be readily extended to other cell formations. $W$ denotes the set of the WiFi APs and we have $|\boldsymbol{W}|=1$. At the same time, the users of the set $\boldsymbol{U}$ are assumed to be uniformly distributed at random in this hybrid VLC/WiFi system. Since each user has a limited FOV, they can only communicate with each other using VLC, if one or more optical APs reside within the FOV of the user. Let $\boldsymbol{R}^{\mathrm{VLC}}$ be the matrix of throughput between the VLC cells $\alpha$ and the user $\mu$, which is defined as $\boldsymbol{R}^{\mathrm{VLC}}=\left(r_{\alpha \mu}: \alpha \in\right.$

${ }^{4}$ In Fig. 2(c), $\hbar$ is given by

$$
\hbar=\frac{1}{2} \cdot \frac{D_{s}}{D_{m c}}+\frac{D_{m c}-D_{s}}{D_{m c}}
$$

where $D_{s}$ and $D_{m c}$ denote the area of the shaded areas within the merged 2 -AP cell and the total area of the merged cell, respectively. 


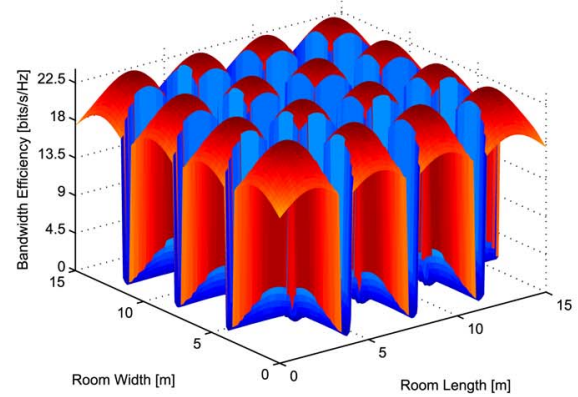

(a) UFR

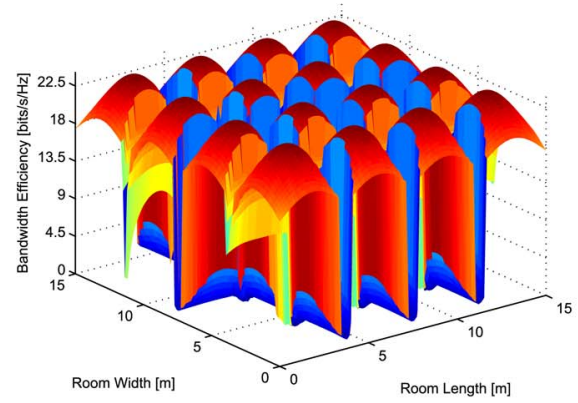

(d) VT-2

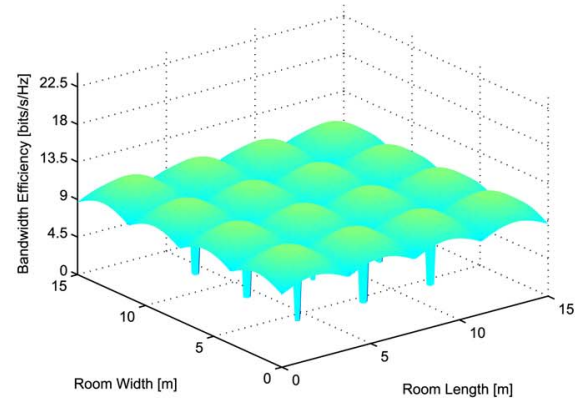

(b) FR-2

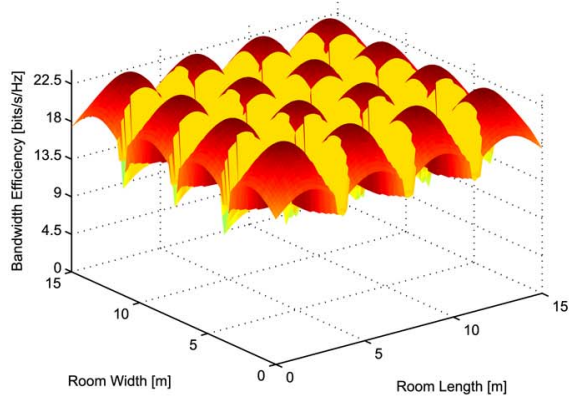

(e) VT-16

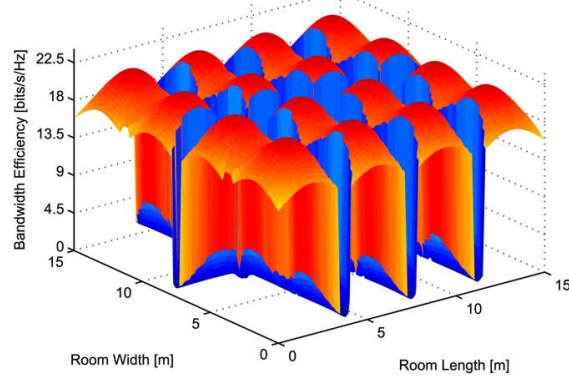

(c) CT-2

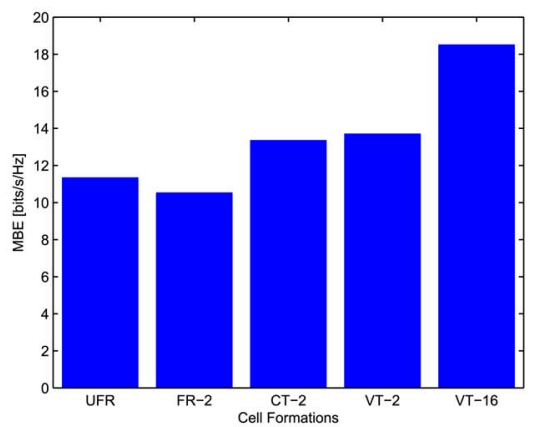

(f) MBE

Fig. 2. BE surface and the MBE of different VLC cell formations. (a) A regular UFR cell formation, (b) has a FR factor of two, (c) represents merged 2-AP cells with CT, (d) shows merged 2-AP cells with VT, (e) is a merged 16-AP cell with VT, and (f) shows the MBE of (a)-(e), where the receivers' FOV is $120^{\circ}$.

$\boldsymbol{C}, \boldsymbol{\mu} \in \boldsymbol{U})$. Let furthermore $\boldsymbol{R}^{\mathrm{WiFi}}=\left(r_{\alpha \mu}: \alpha \in \boldsymbol{W}, \mu \in \boldsymbol{U}\right)$ be the matrix of throughput allocated by the WiFi AP to the user $\mu$.

The LB problem is a connection level issue, which can be performed according to the connection level statistics [20]. Since no fast-fading is experienced in VLC propagation, as long as the users' positions remain static, the channel information does not change. When considering a low-mobility indoor scenario, the coherence time of WiFi propagation is sufficiently long. Hence, both the VLC and WiFi propagation changes slowly in the given period. The required channel information only has to be collected infrequently before activating this optimization procedure. As a result, we consider a static system without the arrival of new users and the departure of existing users. In general, this resorts to the NUM framework [17] and three methodologies will be employed in this section. We commence with an exact non-linear formulation and then discretize this non-linear formulation. Furthermore, we come to the classic distributed algorithm, which is capable of approaching the performance of the near-optimal centralized solution.

\section{A. Centralized Approach}

Our objective is to find the optimal LB using the Objective Function (OF) of maximizing the sum of all users' utility functions under the PF constraints [20] in this hybrid VLC/WiFi system, which is ultimately a joint association control and resource allocation problem. In this context, the authors of [30] have shown that we can achieve proportional allocation by optimizing the OF of:

$$
\operatorname{maximize} \sum_{\mu \in \boldsymbol{U}} \log \left(\beta_{\mu}\right)
$$

where $\beta_{\mu}$ is the actual throughput allocated to a user $\mu$. The logarithm of $\beta_{\mu}$ may be interpreted as the utility function of a user, as argued for example in [31].

At this stage, a binary variable $x_{\alpha \mu}$ is introduced to indicate, whether the user $\mu$ is assigned to the AP $\alpha$, where we have $x_{\alpha \mu}=1$ if $\mu$ and $\alpha$ do have a connection, while 0 otherwise. Note that $\alpha$ may denote either a certain VLC cell or the WiFi $\mathrm{AP}$, namely $\alpha \in \boldsymbol{C} \cup \boldsymbol{W}$ in this section. Hence, the actual throughput $\beta_{\alpha \mu}$ of the user $\mu$ allocated by the AP $\alpha$ may be expressed as $x_{\alpha \mu} r_{\alpha \mu} p_{\alpha \mu}$, where $p_{\alpha \mu}$ is a fractional variable between 0 and 1 , which is denoted as the proportion of time that $\alpha$ is allocated to $\mu$. For a given user $\mu$, the total actual throughput becomes

$$
\beta_{\mu}=\sum_{\alpha \in \boldsymbol{C} \cup \boldsymbol{W}} x_{\alpha \mu} r_{\alpha \mu} p_{\alpha \mu} .
$$

By substituting (15) into the OF of (14), we have

$$
\Gamma(\boldsymbol{x}, \boldsymbol{p})=\sum_{\mu \in \boldsymbol{U}} \log \sum_{\alpha \in \boldsymbol{C} \cup \boldsymbol{W}} x_{\alpha \mu} r_{\alpha \mu} p_{\alpha \mu} .
$$

Since only a single AP $\alpha^{\prime}$ is assigned to a given user, i.e., we have $x_{\alpha^{\prime} \mu}=1$, we can write: $\log \sum_{\alpha} x_{\alpha \mu} r_{\alpha \mu} p_{\alpha \mu}=$ $\sum_{\alpha} x_{\alpha \mu} \log \left(r_{\alpha \mu} p_{\alpha \mu}\right)$. Therefore (16) may equivalently be written as

$$
\Gamma(\boldsymbol{x}, \boldsymbol{p})=\sum_{\mu \in \boldsymbol{U}} \sum_{\alpha \in \boldsymbol{C} \cup \boldsymbol{W}} x_{\alpha \mu} \log \left(r_{\alpha \mu} p_{\alpha \mu}\right) .
$$

Several optimization constraints may be formulated based upon various assumptions as well as on a range of practical 
limitations. The mathematical formulation of this maximization problem becomes:

$$
\begin{array}{ll}
\text { maximize } & \Gamma(\boldsymbol{x}, \boldsymbol{p}) \\
\text { subject to } & \sum_{\alpha \in \boldsymbol{C} \cup \boldsymbol{W}} x_{\alpha \mu}=1 \quad \forall \mu \in \boldsymbol{U} ; \\
& \sum_{\mu \in \boldsymbol{U}} x_{\alpha \mu} p_{\alpha \mu} \leq 1 \quad \forall \alpha \in \boldsymbol{C} ; \\
& \sum_{\mu \in \boldsymbol{U}} x_{\alpha \mu} p_{\alpha \mu} \leq p_{\mathrm{DL}} \quad \forall \alpha \in \boldsymbol{W} ; \\
& x_{\alpha \mu} \in\{0,1\} \quad \forall \alpha \in \boldsymbol{C} \cup \boldsymbol{W}, \forall \mu \in \boldsymbol{U} ; \\
& 0 \leq p_{\alpha \mu} \leq 1 \quad \forall \alpha \in \boldsymbol{C} \cup \boldsymbol{W}, \forall \mu \in \boldsymbol{U} .
\end{array}
$$

Firstly, for each user $\mu$, there is one and only one AP $\alpha^{\prime}$ to satisfy $x_{\alpha^{\prime} \mu}=1$ for an extended period of time. At this stage, we do not impose any constraint on the users' resource demand, hence all the users may either connect to the VLC APs or to the WiFi AP. Secondly, each AP has to request resources confined to its maximum capacity. By contrast, for the DL WiFi AP, the available normalized capacity is assumed to be less than $p_{\mathrm{DL}}$ instead of 1 , because it may allocate $\left(1-p_{\mathrm{DL}}\right)$ of its total resources for example to the uplink. Furthermore, $p_{\mathrm{DL}}$ may be set up as any feasible value between 0 and 1 according to the specific system design and traffic requirements. Finally, the variable $x_{\alpha \mu}$ should be binary, while $p_{\alpha \mu}$ is a real value between 0 and 1. As a result, the above problem represents an MixedInteger Non-Linear Programming (MINLP) problem that may be solved directly by using the OPTI Toolbox, albeit this would be time-consuming.

\section{B. Discretized Linear Programming Approximation}

Instead of solving the MINLP directly, we might opt for a linear relaxation of the original problem. Following the work in [20], we discretize the scheduling time period of each access point into $T$ discrete intervals, where $T$ is $\kappa$ times the number of the users. In practice, $\kappa=10$ turns out to be sufficient to obtain an acceptable approximation of the non-linear problem. A new binary variable $y_{\alpha \mu t}$ is introduced. If and only if the access point $\alpha$ is associated with the user $\mu$ and allocates $t(0 \leq$ $t \leq T)$ time slots to user $\mu$, we arrive at $y_{\alpha \mu t}=1$. Thus, we arrive at the linear OF of

$$
\Gamma_{D}(\boldsymbol{y})=\sum_{\mu \in \boldsymbol{U}} \sum_{\alpha \in \boldsymbol{C} \cup \boldsymbol{W}} \sum_{t=1}^{T} y_{\alpha \mu t} \cdot \log \left(r_{\alpha \mu} \frac{t}{T}\right) .
$$

Accordingly, our linear program is then formulated as

$$
\begin{array}{ll}
\text { maximize } & \Gamma_{D}(\boldsymbol{y}) ; \\
\text { subject to } & \sum_{\alpha \in \boldsymbol{C} \cup \boldsymbol{W}} \sum_{t=1}^{T} y_{\alpha \mu t}=1 \quad \forall \mu \in \boldsymbol{U} ; \\
& \sum_{\mu \in \boldsymbol{U}} \sum_{t=1}^{T} y_{\alpha \mu t} \cdot \frac{t}{T} \leq 1 \quad \forall \alpha \in \boldsymbol{C} ; \\
& \sum_{\mu \in \boldsymbol{U}} \sum_{t=1}^{T} y_{\alpha \mu t} \cdot \frac{t}{T} \leq p_{\mathrm{DL}} \quad \forall \alpha \in \boldsymbol{W} ; \\
y_{\alpha \mu t} \in\{0,1\} \quad \forall \alpha \in \boldsymbol{C} \cup \boldsymbol{W}, \forall \mu \in \boldsymbol{U} .
\end{array}
$$

The first constraint of (20) states that only one and exactly one AP is assigned to each user. The second and third indicate that each AP restricts its time-resource allocation to capacity. This discretized linear problem has been solved with the aid of the CPLEX solver. Then we translate the solution in (20) to the non-linear program (18) as follows:

$$
x_{\alpha \mu}=\sum_{t=1}^{T} y_{\alpha \mu t}, \quad p_{\alpha \mu}=\sum_{t=1}^{T} \frac{t}{T} y_{\alpha \mu t} .
$$

\section{A Dual Decomposition Method}

We now conceive an efficient and scalable distributed algorithm for (18).

1) Transformation: According to [20], when the AP/user association matrix $\boldsymbol{x}$ is given, the unique optimal solution becomes $p_{\alpha \mu}=x_{\alpha \mu} / N_{\alpha}$, where $N_{\alpha}=\sum_{\mu \in \boldsymbol{U}} x_{\alpha \mu}$ is the number of users associated with the access point $\alpha$. As a result, the optimization in (18) may be transformed to a pure association control problem, hence we now have the primal OF of:

$$
\Gamma_{d e}(\boldsymbol{x}, \mathcal{N})=\sum_{\mu \in \boldsymbol{U}} \sum_{\alpha \in \boldsymbol{C} \cup \boldsymbol{W}} x_{\alpha \mu} \log \left(\frac{r_{\alpha \mu}}{N_{\alpha}}\right) .
$$

Additionally, we have three constraints as well:

$$
\begin{aligned}
& \sum_{\mu \in \boldsymbol{U}} x_{\alpha \mu}=N_{\alpha} \quad \forall \alpha \in \boldsymbol{C} \cup \boldsymbol{W} ; \\
& \sum_{\alpha \in \boldsymbol{C} \cup \boldsymbol{W}} x_{\alpha \mu}=1 \quad \forall \mu \in \boldsymbol{U} ; \\
& x_{\alpha \mu} \in\{0,1\} \quad \forall \alpha \in \boldsymbol{C} \cup \boldsymbol{W}, \forall \mu \in \boldsymbol{U} .
\end{aligned}
$$

Furthermore, the dual objective $g(\boldsymbol{\nu})$ is formulated as

$$
g(\boldsymbol{\nu})=\sup _{\boldsymbol{x}, \mathcal{N}} L(\boldsymbol{x}, \mathcal{N}, \boldsymbol{\nu})
$$

where $L(\boldsymbol{x}, \mathcal{N}, \boldsymbol{\nu})$ is the Lagrangian function for (22) using the constraints of (23) after relaxation of (25), yielding

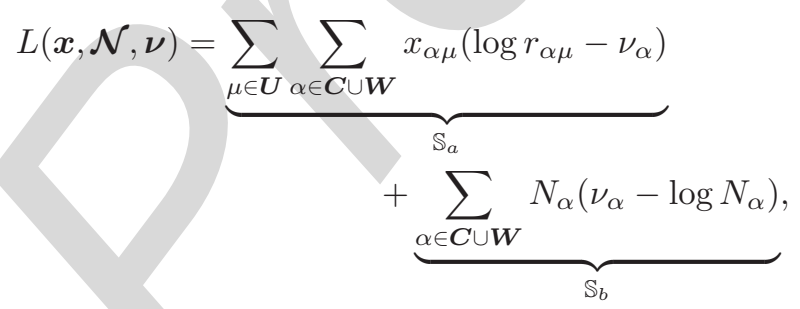

where $\mathcal{N}=\left(N_{\alpha}: \alpha \in \boldsymbol{C} \cup \boldsymbol{W}\right)$ constitutes a set, whose elements are given by the number of users associated with each AP, while $\boldsymbol{\nu}=\left(\nu_{\alpha}: \alpha \in \boldsymbol{C} \cup \boldsymbol{W}\right)$ is the vector of Lagrangian multipliers corresponding to the constraint of (23).

2) Decomposition: The problem of (26) is further partitioned into two sub-problems in a distributive-rather than centralized-fashion.

- On the user's side, we solve the sub-problem of maximizing $\mathbb{S}_{a}$. Since we have $x_{\alpha \mu} \in\{0,1\}$ and $\sum_{\alpha \in \boldsymbol{C} \cup \boldsymbol{W}} x_{\alpha \mu}=1$, for each $\mu \in \boldsymbol{U}$ we find

$$
\alpha^{*}=\underset{\alpha \in \boldsymbol{C} \cup \boldsymbol{W}}{\arg \max }\left(\log r_{\alpha \mu}-\nu_{\alpha}\right) .
$$


Then we set $x_{\alpha^{*} \mu}=1$. Hence we could compute the number of users $\mathcal{N}^{(1)}$ associated with each AP corresponding to $\mathbb{S}_{a}$.

- On the AP's side, upon applying the Karush-Kuhn-Tucker (KKT) conditions [32], for each $\alpha \in \boldsymbol{C} \cup \boldsymbol{W}$ we have

$$
\frac{\partial g^{(2)}}{\partial N_{\alpha}}=\nu_{\alpha}-\log N_{\alpha}+1=0 \Rightarrow N_{\alpha}=\exp \left(\nu_{\alpha}-1\right)
$$

Then we can obtain $\mathcal{N}^{(\mathbf{2})}$ corresponding to $\mathbb{S}_{b}$.

A variable $\delta$ is introduced to reflect the difference between the resource demand $\mathbb{S}_{a}$ and resource supply $\mathbb{S}_{b}$, defined as

$$
\delta=\left|\mathcal{N}^{(1)}-\mathcal{N}^{(2)}\right| .
$$

An acceptable target difference of $\delta_{\text {target }}$ is pre-set before iteratively solving the sub-problems. If each element of $\delta$ is smaller than the target gap, the iterations will be curtailed and the allocation result of $(\hat{\boldsymbol{x}}, \hat{\mathcal{N}})$ is obtained; otherwise $\boldsymbol{\nu}$ will be adjusted for the next iteration according to the gradient method, which is given as

$$
\nu_{\alpha}(i+1)=\nu_{\alpha}(i)-\varepsilon(i)\left(N_{\alpha}-\sum_{\mu \in \boldsymbol{U}} x_{\alpha \mu}\right),
$$

where $\varepsilon(i)$ is a sufficiently small step size in the $i$ th iteration. In this paper, we $\operatorname{set} \varepsilon(i)=\varepsilon_{0} \cdot i^{-\frac{1}{2}+\tau}$, where $\varepsilon_{0}$ and $\tau$ are positive constants appropriately chosen for satisfying $\lim _{i \rightarrow \infty} \varepsilon(i)=$ 0 and $\sum_{i=0}^{i=\infty} \varepsilon(i)=\infty$ for achieving the convergence of the gradient algorithm towards the optimal solution.

\section{Optimality Analysis}

Although the convergence of the distributed algorithm may be proved analytically, e.g. in [25], it is difficult to theoretically study how far the converged solution found is away from that of the true original problem, since the original problem contains a binary integer. Upon comparing the solutions provided by LP approximation, we experimentally study the optimality of the solution provided by the distributed algorithm, which has not been discussed in the literature.

1) Justification: Let us now consider the relationship between the OF value $\Gamma$ of the original MINLP problem (18), the LP OF value $\Gamma_{D}$ for (20) and the solution $\hat{\Gamma}_{d e}$ provided by the distributed algorithm. Since the distributed algorithm is performed after relaxing (25), which prevents us from theoretically proving the optimality of the solution $\hat{\Gamma}_{d e}$ of the problem (22), we opt for an empirical analysis of the optimality of $\Gamma$, $\Gamma_{D}$ and $\hat{\Gamma}_{d e}$.

(i) NLP vs. LP: The authors of [20] have characterized the relationship between the NLP and LP formulations for any feasible solution $(\boldsymbol{y})$ and its corresponding translation $(\boldsymbol{x}, \boldsymbol{p})$, which may be written as

$$
\Gamma_{D} \leq \Gamma \text {. }
$$

(ii) NLP vs. Distributed: We would like to introduce the notation (23') for the relaxation of the problem of (22).

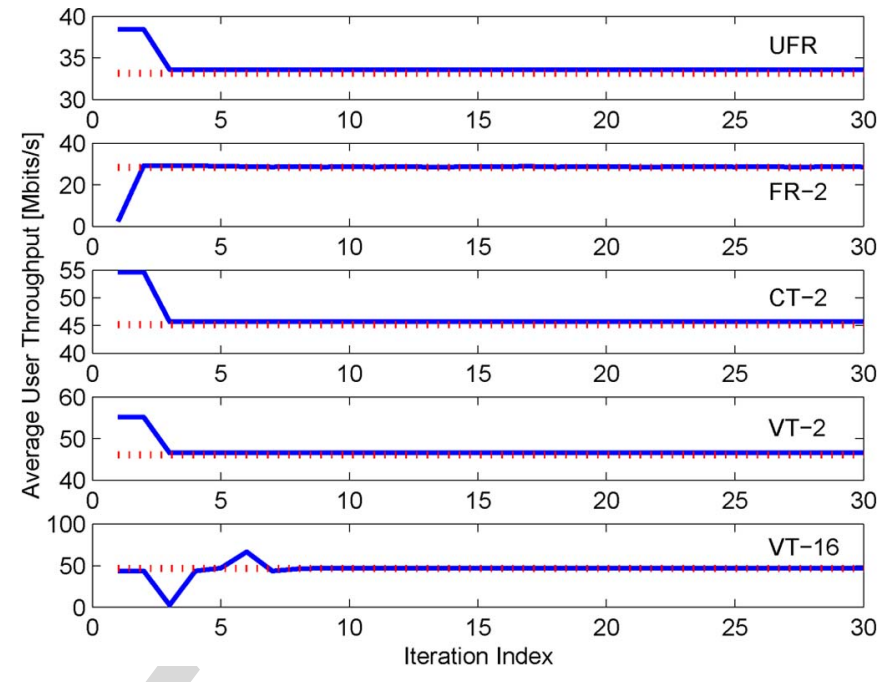

Fig. 3. Empirical optimality of the distributed algorithm relying on the simulation parameters discussed in Section IV-A. The solid line shows the average user throughput in each iteration, while small vertical tick-marks show the solution of the LP problem provided by CPLEX.

Furthermore, we know that $\hat{\Gamma}_{d e}$ upper bounds (23') [32]. Intuitively, $\hat{\Gamma}_{d e}$ may also upper bound (22), since the solution of $x_{\alpha \mu}$ always satisfies the constraint (25) in the process of solving (23').

(iii) NLP, LP and Distributed: Combing (i) and (ii) above, for any feasible pair, we may use $\hat{\Gamma}_{d e}$ and $\Gamma_{D}$ as the upper bound and the lower bound for the original MINLP problem, respectively. Please note that all the above discussions are here related to the resource allocation lemma detailed at the beginning of Section III-C [20]. Under this lemma (18) may be written as (22).

Fig. 3 shows the empirical optimality of our distributed algorithm with the aid of simulations relying on the parameters discussed in Section IV-A. The discretized LP approximation is implemented in our simulation scenarios and the near-optimal solution of the LP OF is shown by the dashed line in Fig. 3. We obtain a fairly accurate solution of the distributed OF within a dozen of iterations, which is within a margin of about $1.5 \%$ from the sub-optimal LP solution. Since we may derive from (iii) that the optimal NLP OF value $\Gamma$ may be between $\Gamma_{D}$ and $\hat{\Gamma}_{d e}$, the difference between $\hat{\Gamma}_{d e}$ and $\Gamma$ may be even smaller. This demonstrates that the distributed algorithm is capable of converging to the optimal value of the original NLP problem, whilst providing a near-optimal solution within a few dozens of iterations.

2) Implementation: As a benefit of the above optimality analysis, we opt for the distributed algorithm. Indeed, the distributed algorithm converges to a near-optimal solution significantly faster than the centralized approaches. In each iteration, each AP initializes and broadcasts a feasible price value $\nu_{\alpha}$ to all the users within its coverage. Here we assume that different APs use pre-allocated orthogonal frequency bands for simultaneously broadcasting $\nu_{\alpha}$. Each user finds the optimal AP $\alpha^{*}$ according to (28) and sends its user-identifier back to $\alpha^{*}$. Thus each AP becomes capable of calculating the user demand $\mathcal{N}^{(1)}$. At the same time, the supply vector $\mathcal{N}^{(2)}$ can also be calculated by the APs. Each AP compares its supply and user 
demand. When the difference between the demand and supply becomes sufficiently small, the iterations are curtailed and a near-optimal solution has been found. In this way, each AP becomes capable of performing its own resource allocation. The total number of information exchange operations is proportional to $\left(n_{\alpha}+n_{\mu}\right)$, where $n_{\alpha}$ and $n_{\mu}$ denote the number of APs and users, respectively. Let us now provide an overview of the dual decomposition algorithm in form of Algorithm 1, which has been verified using our simulations.

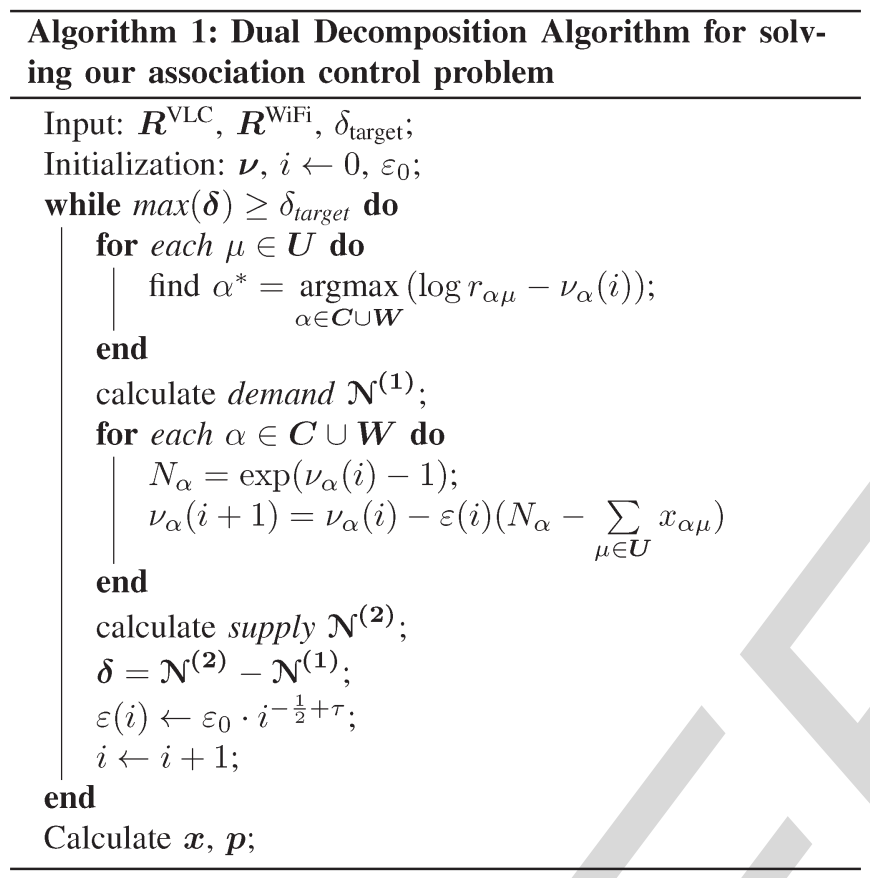

\section{Performance Evaluations}

In this section, we will present our simulation results for the LB problem, with special emphasis on the aforementioned various VLC cell formations in our hybrid VLC/WiFi system. We commence by studying the user's average throughput for different FOV and LOS blocking probability, followed by investigating the fairness both from the system's and the individual users' perspectives for the sake of characterizing the quality of service experienced by the users under different cell formation schemes.

\section{A. Simulation Setup}

A $15 \mathrm{~m} \times 15 \mathrm{~m} \times 3 \mathrm{~m}$ room model is considered, which is only covered by a VLC system including $4 \times 4$ uniformly distributed optical APs at a height of $2.5 \mathrm{~m}$. Additionally, the room is entirely covered by an IEEE Std 802.11n WiFi AP supporting a data rate as high as 120 Mbits/s within $25 \mathrm{~m}$. The parameters of the LED lamps are summarized in Table I. The normalized WiFi DL capacity $p_{\mathrm{DL}}$ is assumed to be 0.8 and $U=50$ users are assumed to be distributed uniformly at random during each simulation run. All of the results shown are averaged over 50 simulation runs. To be more realistic, we introduce specific modulation schemes for calculating the users' achievable throughput. For simplicity, we consider base-

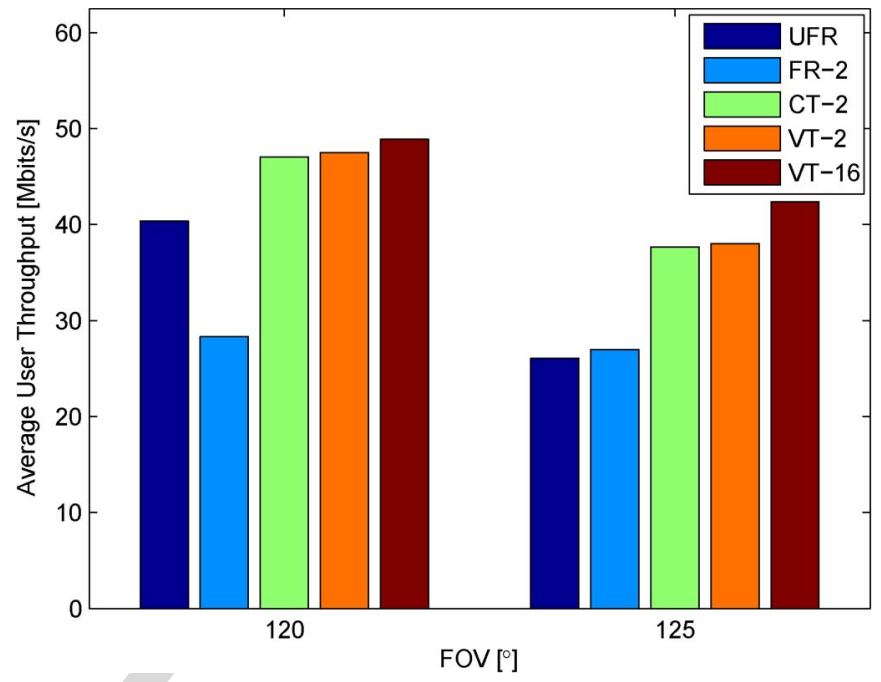

Fig. 4. Average user throughput of various cell formations with different FOV after performing LB in the hybrid VLC/WiFi system.

band transmissions without subcarrier modulation at this stage. Pulse-Amplitude Modulation having an order of $M$ ( $M$-PAM) is used. Based on our Bit Error Rate (BER) performance results, given a certain target BER, Signal to Noise Ratio (SNR) and Signal to Interference Ratio (SIR), the maximum affordable $M$-PAM order capable of maintaining the target-BER can be determined. ${ }^{5}$ Thus the attainable throughput becomes $r_{\alpha \mu}=$ $2 B \log _{2} M /(1+\rho)$, where the roll-off factor of the raisedcosine pulses is assumed to be $\rho=1$.

\section{B. Throughput Investigations}

1) Throughput Investigations for Different FOV: The FOV is one of the factors that is expected to significantly influence the ICI in VLC-based networks. Increasing the FOV leads to the expansion of the ICI-contaminated areas and correspondingly the employment of ICI reduction techniques may become more important. Fig. 4 shows the average user throughput of different cell formations with different FOV after performing LB in the hybrid VLC/WiFi system. We can see that the throughput provided by the merged cells is higher than the regular cell formations for both FOV values. Since the increase of FOV may result in 1) an increased probability of having user in the ICIcontaminated areas and 2) an decease of received signal power and an increase of received noise power, the average throughput is reduced in all cell formation scenarios for FOV $=120^{\circ}, 125^{\circ}$. In particular, the UFR scheme is the most badly affected one upon increasing the FOV, which supports the lowest throughput, when the FOV is increased to $125^{\circ}$. Furthermore, FR-2 does not change much.

\footnotetext{
${ }^{5}$ We could also consider the joint effects of noise and interference. According to [5], the relationship between the BER and $\xi$ for $M$-PAM signals is given by

$$
\mathrm{BER}_{M-\mathrm{PAM}} \cong \frac{M-1}{M} \frac{2}{\log _{2} M} Q\left(\frac{\sqrt{\xi}}{M-1}\right) .
$$

Given a certain target BER and the above-mentioned SINR value $\xi$, this is an alternative technique of determining the maximum PAM order $M$, which agrees with our simulation results.
} 

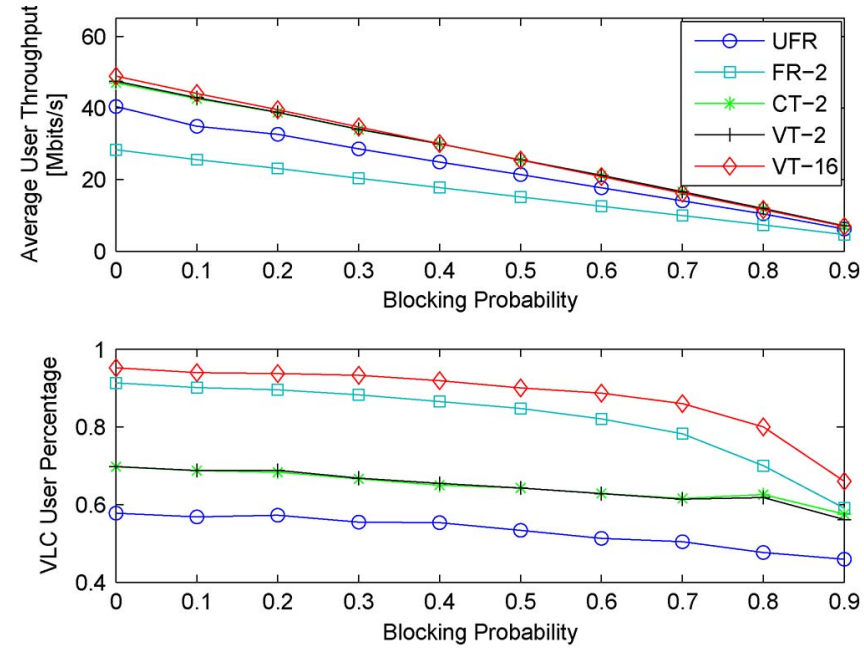

Fig. 5. Average user throughput and proportion of VLC users under various LOS blocking probabilities, FOV $=120^{\circ}$.

2) LOS Blocking Analysis: As mentioned in Section I, the WiFi AP is also capable of providing seamless DL coverage, when the LOS VLC-DL receptions are blocked. We introduce the LOS blocking probability $P_{\text {block }}$ to represent the probability that the LOS VLC path is blocked, which may lead to a reduction of the data rate experienced by some VLC users. Then the VLC DL data rate becomes $\widetilde{R}=P_{\text {block }} \cdot 0+\left(1-P_{\text {block }}\right) \cdot R$. At this stage, we assume that all LOS paths are blocked with an equal probability. Fig. 5 indicates that, as expected, the average user throughput and the proportion of VLC users is reduced upon increasing the LOS blocking probability in all cell formation scenarios, hence more users are allocated to the WiFi AP. This demonstrates that the WiFi AP plays an important role in this hybrid system, especially when the LOS paths may be blocked.

\section{Fairness Grade Investigations}

1) Average Fairness: Next we will analyse both the network's average fairness as well as the individual user's fairness, given a certain total throughput. The Grade of Fairness (GOF) for the network is defined as

$$
\sigma_{a-\mathrm{VLC}}=\left|1-\frac{\mathrm{VLC}-\text { fractionof total throughput }}{\text { fraction of VLC }- \text { users }}\right|
$$

For example, in the UFR scenario, the VLC throughput proportion of the total VLC/WiFi throughput is $95.25 \%$, while $57.76 \%$ of the users is supported by the VLC APs, rather than by WiFi. Hence the grade of the average fairness becomes $\sigma_{a}=\mid 1-$ $95.25 \% / 57.76 \% \mid \approx 0.649$. The system provides the highest grade of average fairness, when the VLC (WiFi) throughput accounts for a certain proportion of the total throughput and at the same time, the number of the VLC (WiFi) users accounts for the same proportion of the total number of users in the hybrid system. Hence for the highest possible GOF we have $\sigma_{a}=0$. It is plausible that the system will provide a higher grade of average fairness, when $\sigma_{a}$ is closer to zero, provided that no

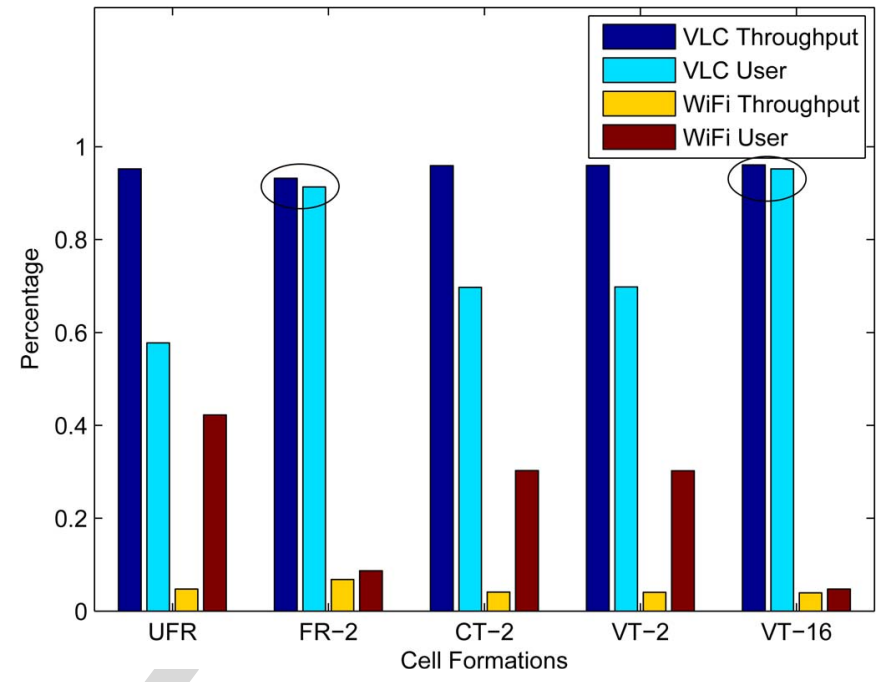

Fig. 6. Throughput and user percentage in different cell formations, FOV $=$ $120^{\circ}$

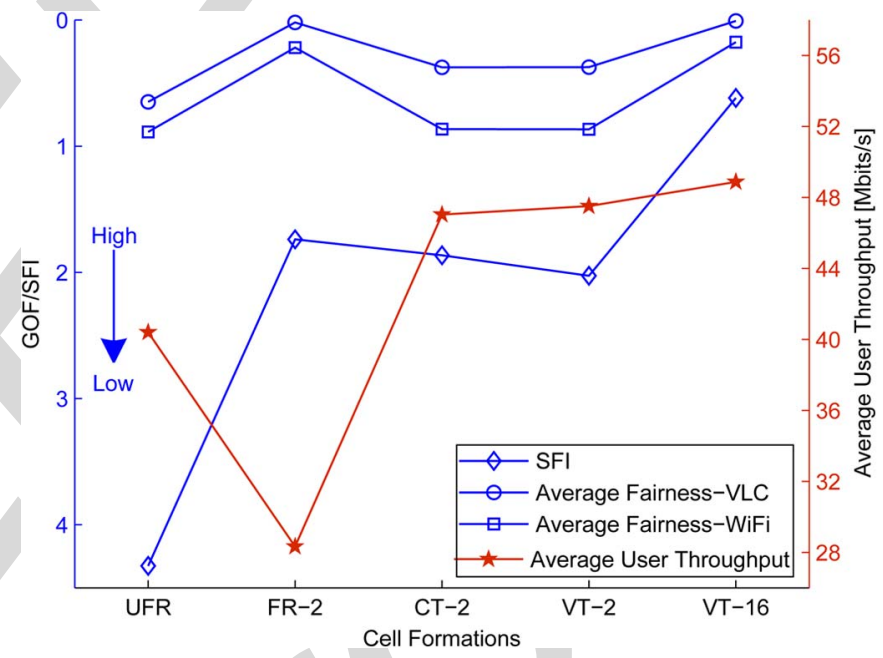

Fig. 7. The GOF, SFI and average user throughput, FOV $=120^{\circ}$. The $\mathrm{GOF} / \mathrm{SFI}$ perceived is reduced upon increasing its value.

multi-service requirements are considered at this stage [26]. Since the sum of VLC throughput (users) and WiFi throughput (users) constitutes the total throughput (users) of the system, the VLC system and the WiFi system provide a similar grade of average fairness. When the difference between the VLC (WiFi) throughput proportion and the fraction of VLC (WiFi) users is smaller, $\sigma_{a}$ is also reduced, i.e. the hybrid system provides a higher grade of average fairness.

We know that the size of ICI-contaminated areas is significantly smaller when the FR factor is two and no ICI occurs when all the VLC APs are merged into a 16-AP cell employing VT. Hence more users are expected to be supported by VLC APs in these two scenarios compared to the UFR, CT-2 and VT-2 schemes. We can see from Fig. 6 that the VLC (WiFi) network throughput and the corresponding user-proportion are closest to each other, since the FR-2 and VT-16 scenario have a higher percentage of VLC users, as indicated by the circles in Fig. 6. Specific values of $\sigma_{a}$ are plotted in Fig. 7. 


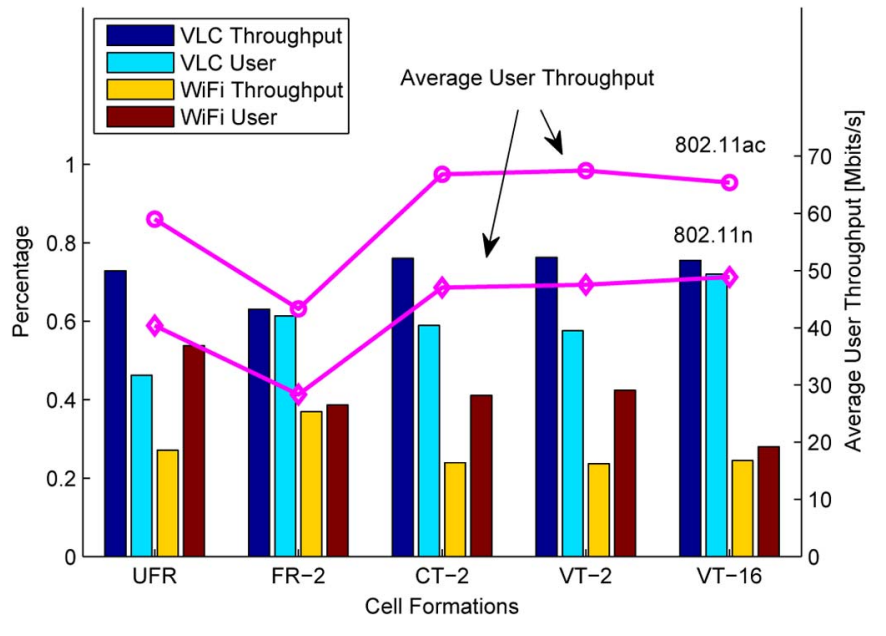

Fig. 8. Throughput and user percentage in different cell formations, FOV = $120^{\circ}$. WiFi data rate is set to $1 \mathrm{Gbits} / \mathrm{s}$.

2) Individual Fairness: We will use the Service Fairness Index (SFI) of [33] to reflect the individual fairness experienced by the users. The objective of ensuring fairness amongst the users is to guarantee that all users benefit from the same throughput within a given period, provided that the users' data rate requirements are identical. However, this is often unrealistic. The SFI of [33] is introduced to represent the difference between the maximum and the minimum amongst all the users' throughput. When the SFI is low, the throughput-difference of different users is small, hence they are served fairly. By contrast, if the SFI is high, the users experiencing a lower data rate may complain about their unfair treatment. Therefore a lower SFI means a higher grade of individual fairness. The specific SFI values of the different cell formation scenarios considered are plotted in Fig. 7. It can be clearly seen that the users' throughput-difference is the smallest in the VT-16 scheme, which is expected on the basis of Fig. 2(e), where VT-16 provides the smoothest BE distribution.

Considering the average user throughput and fairness performance comprehensively in Fig. 7, the merged 16-AP cell relying on VT is the most attractive one having the highest throughput as well as the highest GOF and SFI. However, its implementation complexity is also significantly higher than that of the regular designs and of CT.

\section{1 Gbis/s-Data-Rate WiFi}

Since 802.11ac may support a data rate over $1 \mathrm{Gbits} / \mathrm{s}$, it is realistic to investigate a hybrid system having a WiFi data rate of 1 Gbits/s. Fig. 8 shows the average user throughput, the throughput and user percentages in the different cell formations having a WiFi data rate as high as $1 \mathrm{Gbits} / \mathrm{s}$ and $\mathrm{FOV}=120^{\circ}$. The average user throughput is increased in all the scenarios considered. Both the percentage share of WiFi throughput and the proportion of its users are increased with the improvement of the WiFi data rate. The merged cells still provide a higher throughput, however the single 16-AP cell relying on VT may no longer have the absolute advantage in terms of its average throughput. This may provide some insights for this hybrid systems' design, when the WiFi data rate is increased.

\section{CONCLUSiON}

In this paper, various VLC cell formation schemes and a heterogeneous system constituted by WLANs and VLC networks were investigated. We studied the regular design concept borrowed from cellular networks relying on different FR factors in VLC environments as well as of merged multi-AP cells employing either CT or ZF-based VT. To solve the essential LB problem in the context of our VLC/WiFi hybrid system, both centralized and distributed algorithms were invoked for implementing a PF scheduler. We analysed the MBE of different VLC cell formations as well as the throughput and fairness of the hybrid VLC/WiFi system. By employing a sophisticated VT among all the 16 VLC APs, the VLC network becomes capable of providing a higher MBE, while the hybrid system is capable of providing a higher average throughput without any sacrifice of the fairness, when the WiFi data rate is modest.

\section{REFERENCES}

[1] L. Hanzo et al., "Wireless myths, realities, futures: From 3G/4G to optical and quantum wireless," in Proc. IEEE, May 2012, vol. 100, pp. 1853-1888.

[2] H. Elgala, R. Mesleh, and H. Haas, "Indoor optical wireless communication: Potential and state-of-the-art," IEEE Commun. Mag., vol. 49, no. 9, pp. 56-62, Sep. 2011.

[3] J. Kahn and J. Barry, "Wireless infrared communications," Proc. IEEE, vol. 85, no. 2, pp. 265-298, Feb. 1997.

[4] Z. Ghassemlooy, W. Popoola, and S. Rajbhandari, Optical Wireless Communications: System and Channel Modelling With MATLAB. Boca Raton, FL, USA: CRC Press, 2012.

[5] J. Grubor, S. Randel, K.-D. Langer, and J. Walewski, "Broadband information broadcasting using led-based interior lighting," J. Lightw. Technol., vol. 26, no. 24, pp. 3883-3892, Dec. 2008.

[6] T. Komine and M. Nakagawa, "Fundamental analysis for visible-light communication system using led lights," IEEE Trans. Consum. Electron., vol. 50, no. 1, pp. 100-107, Feb. 2004.

[7] S. Dissanayake and J. Armstrong, "Comparison of ACO-OFDM, DCO-OFDM and ADO-OFDM in IM/DD systems," J. Lightw. Technol., vol. 31, no. 7, pp. 1063-1072, Apr. 2013.

[8] X. Li, J. Vucic, V. Jungnickel, and J. Armstrong, "On the capacity of intensity-modulated direct-detection systems and the information rate of ACO-OFDM for indoor optical wireless applications," IEEE Trans. Commun., vol. 60, no. 3, pp. 799-809, Mar. 2012.

[9] R. Mesleh, H. Elgala, and H. Haas, "On the performance of different OFDM based optical wireless communication systems," IEEE/OSA J. Opt. Commun. Netw., vol. 3, no. 8, pp. 620-628, Aug. 2011.

[10] J. Armstrong, "OFDM for optical communications," J. Lightw. Technol., vol. 27, no. 3, pp. 189-204, Feb. 2009.

[11] H. Le-Minh et al., "100-Mb/s NRZ visible light communications using a postequalized white LED," IEEE Photon. Technol. Lett., vol. 21, no. 15, pp. 1063-1065, Aug. 2009.

[12] IEEE Standard for Local and Metropolitan Area Networks-Part 15.7: Short-Range Wireless Optical Communication Using Visible Light, IEEE Std 802.15.7-2011, Sep. 2011, pp. 1-309.

[13] M. Rahaim, A. Vegni, and T. D. C. Little, "A hybrid radio frequency and broadcast visible light communication system," in Proc. IEEE GLOBECOM Workshops, Dec. 2011, pp. 792-796.

[14] Y. Wang, S. Videv, and H. Haas, "Dynamic load balancing with handover in hybrid Li-Fi and Wi-Fi networks," in Proc. IEEE 25th Int. Symp. Pers., Indoor Mobile Radio Commun., Washington, DC, USA, 2014.

[15] C. Chen, N. Serafimovski, and H. Haas, "Fractional frequency reuse in optical wireless cellular networks," in Proc. IEEE PIMRC, Sep. 2013, pp. 3594-3598.

[16] C. Chen, D. Tsonev, and H. Haas, "Joint transmission in indoor visible light communication downlink cellular networks," in Proc. IEEE GLOBECOM Workshops, Dec. 2013, pp. 1127-1132.

[17] D. Palomar and M. Chiang, "A tutorial on decomposition methods for network utility maximization," IEEE J. Sel. Areas Commun., vol. 24, no. 8, pp. 1439-1451, Aug. 2006. 
[18] J. Andrews, S. Singh, Q. Ye, X. Lin, and H. Dhillon, "An overview of load balancing in hetnets: Old myths and open problems," IEEE Wireless Commun., vol. 21, no. 2, pp. 18-25, Apr. 2014.

[19] T. Bu, L. Li, and R. Ramjee, "Generalized proportional fair scheduling in third generation wireless data networks," in Proc. INFOCOM, Apr. 2006, pp. 1-12.

[20] L. Li, M. Pal, and Y. Yang, "Proportional fairness in multi-rate wireless LANs," in Proc. INFOCOM, Apr. 2008, pp. 1-9.

[21] K. Son, S. Chong, and G. Veciana, "Dynamic association for load balancing and interference avoidance in multi-cell networks," IEEE Trans. Wireless Commun., vol. 8, no. 7, pp. 3566-3576, Jul. 2009.

[22] H. Burchardt, S. Sinanovic, Z. Bharucha, and H. Haas, "Distributed and autonomous resource and power allocation for wireless networks," IEEE Trans. Commun., vol. 61, no. 7, pp. 2758-2771, Jul. 2013.

[23] F. Heliot, M. Imran, and R. Tafazolli, "Low-complexity energy-efficient resource allocation for the downlink of cellular systems," IEEE Trans. Commun., vol. 61, no. 6, pp. 2271-2281, Jun. 2013.

[24] M. Ismail and W. Zhuang, "A distributed multi-service resource allocation algorithm in heterogeneous wireless access medium," IEEE J. Sel. Areas Commun., vol. 30, no. 2, pp. 425-432, Feb. 2012.

[25] Q. Ye et al., "User association for load balancing in heterogeneous cellular networks," IEEE Trans. Wireless Commun., vol. 12, no. 6, pp. 2706-2716, Jun. 2013.

[26] F. Jin, R. Zhang, and L. Hanzo, "Resource allocation under delayguarantee constraints for heterogeneous visible-light and RF femtocell," IEEE Trans. Wireless Commun. (Accepted), vol. 14, Feb. 2015.

[27] F. R. Gfeller and U. Bapst, "Wireless in-house data communication via diffuse infrared radiation," Proc. IEEE, vol. 67, no. 11, pp. 1474-1486, Nov. 1979.

[28] H. Zhang and H. Dai, "Cochannel interference mitigation and cooperative processing in downlink multicell multiuser MIMO networks," EURASIP J. Wireless Commun. Netw., vol. 2004, no. 2, pp. 222-235, Dec. 2004.

[29] R. Zhang and L. Hanzo, "Cooperative downlink multicell preprocessing relying on reduced-rate back-haul data exchange," IEEE Trans. Veh. Technol., vol. 60, no. 2, pp. 539-545, Feb. 2011.

[30] F. Kelly, "Charging and rate control for elastic traffic," Eur. Trans. Telecommun., vol. 8, no. 1, pp. 33-37, Jan./Feb. 1997.

[31] J. Akhtman and L. Hanzo, "Power versus bandwidth-efficiency in wireless communications: The economic perspective," in Proc. IEEE VTC, Sep. 2009, pp. 1-5.

[32] S. P. Boyd and L. Vandenberghe, Convex Optimization. Cambridge, U.K.: Cambridge Univ. Press, 2004

[33] B. Bensaou, D. H. K. Tsang, and K. T. Chan, "Credit-based fair queueing (CBFQ): A simple service-scheduling algorithm for packet-switched networks," IEEE/ACM Trans. Netw., vol. 9, no. 5, pp. 591-604, Oct. 2001.

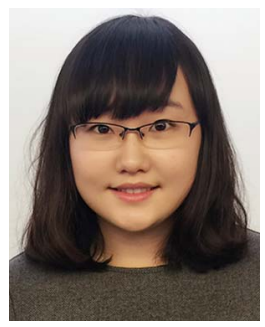

Xuan Li received the B.Eng. degree in optical information science and technology from Beijing Institute of Technology, China, in 2012. She is currently working toward the Ph.D. degree with the Southampton Wireless Group, University of Southampton, UK. Her research interests include visible light communications, heterogeneous networks, resource allocation, and scheduling algorithms.

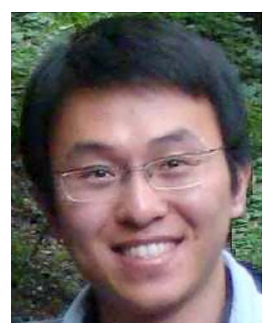

Rong Zhang (M'09) received the B.Sc. degree from Southeast University, China, in 2003, and the Ph.D. degree from Southampton University, UK, in 2009. Before receiving the doctorate, he was an Engineer (August 2003-July 2004) at China Telecom and a Research Assistant (January 2006-May 2009) at Mobile Virtual Center of Excellence (MVCE), U.K. After being a Post-doctoral Researcher (August 2009-July 2012) at Southampton University, he took industrial consulting leave (August 2012-January 2013) for Huawei Sweden R\&D as a System Algorithms Specialist. Since February 13, he has been an appointed as a Lecturer at CSPC group of ECS, Southampton University. He has 40+ journals in prestigious publication avenues (e.g. IEEE, OSA) and many more in major conference proceedings. He regularly serves as reviewer for IEEE transactions and journals and has been a TPC Member/Invited Session Chair of major conferences. He is the recipient of joint funding of MVCE and EPSRC and is also a Visiting Researcher under Worldwide University Network (WUN). More details can be found at http://www.ecs.soton.ac.uk/people/rz

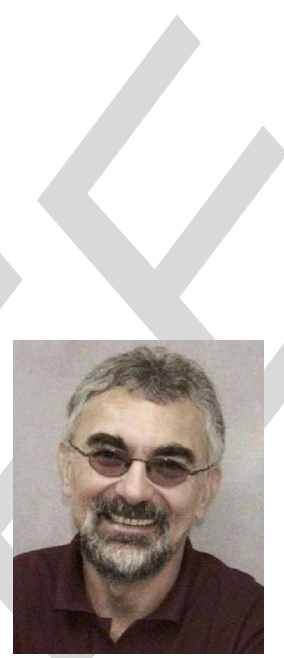

Lajos Hanzo received the bachelor's degree in electronics in 1976 and the doctorate degree in 1983. In 2009 he was awarded the Doctor Honoris Causa by the Technical University of Budapest. During his 38-year career in telecommunications, he has held various research and academic posts in Hungary, Germany, and the U.K. Since 1986 he has been with the School of Electronics and Computer Science, University of Southampton, U.K, where he holds the chair in telecommunications. He has successfully supervised about $100 \mathrm{Ph} . \mathrm{D}$. students, co-authored 20 John Wiley/IEEE Press books on mobile radio communications totalling in excess of 10000 pages, published over 1400 research entries at IEEE Xplore, acted both as TPC and General Chair of IEEE conferences, presented keynote lectures and has been awarded a number of distinctions. Currently, he is directing a 100-strong academic research team, working on a range of research projects in the field of wireless multimedia communications sponsored by industry, the Engineering and Physical Sciences Research Council (EPSRC) U.K., the European Research Council's Advanced Fellow Grant and the Royal Society's Wolfson Research Merit Award. He is an enthusiastic supporter of industrial and academic liaison and he offers a range of industrial courses. $\mathrm{He}$ is also a Governor of the IEEE Vehicular Technology Society. From 2008 to 2012, he was the Editor-in-Chief of the IEEE Press and a Chaired Professor at Tsinghua University, Beijing, China. His research is funded by the European Research Council's Senior Research Fellow Grant. For further information on his research in progress and associated publications please refer to http:// www-mobile.ecs.soton.ac.uk. 


\title{
Cooperative Load Balancing in Hybrid Visible Light Communications and WiFi
}

\author{
Xuan Li, Rong Zhang, Member, IEEE, and Lajos Hanzo
}

\begin{abstract}
As a complementary extension of established Radio Frequency (RF) Wireless Local Area Networks (WLANs), Visible Light Communication (VLC) using commercially available Light-Emitting Diode (LED) transmitters offers a huge data rate potential in this license-free spectral domain, whilst simultaneously satisfying energy-efficient illumination demands. Various VLC cell formations, ranging from a regular cell-layout associated with different Frequency Reuse (FR) patterns to merged cells by employing advanced transmission scheme are investigated. Furthermore, a hybrid Down-Link (DL) offering full RF-coverage by a WLAN and additionally supported by the abundant spectral resources of a VLC network is studied. Cooperative Load Balancing (LB) achieving Proportional Fairness (PF) is implemented by using both centralized and distributed resource-allocation algorithms. The performance of this hybrid RF/VLC system is analysed both in terms of its throughput and fairness in diverse cell formation scenarios. Our simulation results demonstrate that, the VLC system advocated is capable of providing a high Area Spectral Efficiency (ASE) and our hybrid RF/VLC system achieves the highest throughput and the highest grade of fairness in most of the scenarios considered.
\end{abstract}

Index Terms-Cell formation, cooperative load balancing, heterogeneous networks, visible light communication.

\section{INTRODUCTION}

W ITH the promise of gaining access to a huge unlicensed bandwidth, which is available in the optical domain of the electromagnetic spectrum, the research of Optical Wireless (OW) communications intensified during the past decade or so [1]. Apart from the substantial amount of research on the infrared region of the optical spectrum [2], [3], as a benefit of the rapid development of solid-state lighting, high data rate Visible Light Communication (VLC) combined with advanced illumination has become a reality in indoor scenarios [4]-[6]. Specifically, the Light-Emitting Diodes (LEDs) exhibit a high energy efficiency and additionally they are capable of exploiting a vast unregulated spectrum. Extensive investigations have

Manuscript received November 15, 2013; revised May 1, 2014, October 1, 2014, and January 14, 2015; accepted February 24, 2015. The financial support of the RC-UK under the auspices of the UK-India ATC in Wireless Communications, of the Chinese Scholarship Council as well as of the European Research Council's Advanced Fellow Grant is gratefully acknowledged. The associate editor coordinating the review of this paper and approving it for publication was H. Haas.

The authors are with the Communications, Signal Processing and Control, School of ECS, University of Southampton, Southampton SO17 1BJ, U.K. (e-mail: x126g12@ecs.soton.ac.uk; rz@ecs.soton.ac.uk; 1h@ecs.soton.ac.uk).

Color versions of one or more of the figures in this paper are available online at http://ieeexplore.ieee.org.

Digital Object Identifier 10.1109/TCOMM.2015.2409172 been dedicated to the physical layer of VLC [7]-[11], as also indicated by the IEEE 802.15.7 standard ratified for shortrange visible light wireless communication [12]. As far as the network level of our VLC system is concerned, stand-alone VLC networks may exhibit some potential drawbacks, such as for example: 1) VLC networks perform poorly in nonline-of-sight scenarios owing to the predominantly Line-OfSight (LOS) propagation of light; 2) In VLC networks, each optical Access Point (AP) illuminates only a small confined cell compared to cellular RF networks; 3) VLC networks fail to provide convenient Up-Link (UL) coverage at the current-stateof-the-art. To overcome the above drawbacks, it is necessary to develop cooperative Heterogeneous Networks (HetNets), which additionally rely on Radio Frequency (RF) techniques as a complementary extension. As a result, the widely used Wireless Fidelity (WiFi) network may be invoked as a cooperative partner of the VLC networks in indoor scenarios. As mentioned above, the proposed hybrid VLC/WiFi system is capable of providing high-data-rate connections as well as a seamless reliable coverage [1]. Compared to the traditional WiFi-only and VLC-only systems, the integration of VLC and WiFi is expected to significantly improve the aggregate throughput, which has been shown both by analytical and simulation results in [13] and the independent efforts disseminated in [14] led to similar conclusions. This motivates our research of the hybrid VLC/WiFi system by investigating fair and efficient cooperative Load Balancing (LB), where a salient problem is the appropriate formation of VLC cells.

There is a paucity of studies on the formulation of VLC cells, although recently some valuable studies were disseminated in the context of a stand-alone VLC-only system. In particular, the authors of [15] discussed Fractional Frequency Reuse (FFR) for VLC cells and subsequently a joint transmission regime was derived in [16] for VLC cells. ${ }^{1}$ On the other hand, LB constitutes one of the fundamental problems, which in fact affects all cooperative multi-rate HetNets. Broadly speaking, this problem can be formulated as the constrained optimization of a carefully selected utility function [17]. Substantial related work has been undertaken based on this problem in RF networks [18]-[25]. Specifically, the authors of [18] investigated diverse technical approaches to the LB problems of HetNes and provided valuable design guidelines for OFDMA-based cellular systems. Moreover, the authors of [19]-[21] proposed centralized solutions, which rely on a centralized resource manager,

\footnotetext{
${ }^{1}$ Our combined transmission proposed in this article is reminiscent of the joint transmission introduced in [16], which was published after our submission. Hence these two works are independently developed.
} 
while the authors of [22]-[25] addressed the LB problems of cellular networks with the aid of distributed algorithms. More particularly, Burchardt et al. [22] introduced a fuzzy logic based system, while Heliot et al. [23] proposed the employment of the Newton-Raphson-based method. However, both of them considered a homogeneous single-network scenario, rather than a VLC/RF HetNet scenario. As a further advance, in [24], [25] the LB problem of a RF-based HetNet was solved by using the dual decomposition approach and provided a near-optimal solution at a low complexity. However, the LB problem of VLC-based HetNets has remained to a large extent hitherto unexplored [26], especially when combined with various VLC cell formations.

Against the above-mentioned background, in this paper, we investigate the LB problem between several VLC APs and a WiFi AP relying on the users' Proportional Fairness (PF) as a measure, when jointly considering various VLC cell formations, ranging from traditional cellular design to merged cells.

The rest of this paper is organized as follows. Our hybrid system model and the various cell formations considered are presented in Section II.

Our methodology for solving this LB problem, including both the centralized as well as its decomposed/distributed counterpart are described and evaluated in Section III and Section IV, respectively. Finally, Section V offers our conclusions.

Notation: Bold variables are used to denote vectors, matrices or sets, while regular Roman characters are used for general parameters.

\section{SYSTEM MODEL}

A hybrid VLC/WiFi Down-Link (DL) system model is considered, where the IEEE 802.11 Wireless Local Area Network (WLAN) is complemented by an optical network. The hybrid network has a set of VLC APs as well as a WiFi AP, but this scenario may be readily extended to other AP configurations. More explicitly, each VLC AP relies on an LED lamp constructed from several LEDs. Let us first discuss a range of VLC cell formations in this section before investigating our LB problems.

\section{A. Link Characteristic}

For a given transmitted optical power $P_{t}$ of each VLC AP, the average optical power $P_{r}$ received by a photodetector is the sum of the power received from all the corresponding transmitting VLC APs within its vicinity, which is hosted in the set $\boldsymbol{S}$ and it is given as ${ }^{2}$

$$
P_{r}=\sum_{i \in S} P_{r, i}=\sum_{i \in S} H_{i}(0) P_{t}
$$

\footnotetext{
${ }^{2}$ Since our major concern is that of investigating various VLC cell formations and finding an efficient LB solution for this hybrid system, some of the practical VLC channel characteristics have been simplified. The optical channel of (2) may be widely adopted, when considering a Lambertian source in indoor optical wireless scenarios. Our algorithm is a generic one, which may be readily adapted to other types of optical channels.
}

TABLE I

VLC PARAMETERS

\begin{tabular}{l|r}
\hline \hline Transmitted optical power per LED lamp $\left(P_{t}\right)$ & $20[\mathrm{~W}]$ \\
Semi-angle at half power $\left(\phi_{1 / 2}\right)$ & $60^{\circ}$ \\
Modulation bandwidth $(B)[4],[5],[9],[11]$ & $20[\mathrm{MHz}]$ \\
Physical area of a PD $\left(D_{\mathrm{PA}}\right)$ & $1.0\left[\mathrm{~cm}^{2}\right]$ \\
Gain of an optical filter $\left(T_{s}(\psi)\right)$ & 1.0 \\
Refractive index of a lens at a PD $(n)$ & 1.5 \\
O/E conversion efficiency $(\gamma)$ & $0.53[\mathrm{~A} / \mathrm{W}]$ \\
Half of the receiver's FOV $\left(\psi_{\mathrm{F}}\right)$ & $60^{\circ} / 65^{\circ}$ \\
BER threshold & $10^{-5}$ \\
Roll-off factor $(\rho)$ & 1 \\
Normalized WiFi capacity for DL $\left(p_{\mathrm{DL}}\right)$ & 0.8 \\
Supply and demand gap $\left(\delta_{\text {target }}\right)$ & 1 \\
\hline \hline
\end{tabular}

According to [3], [27], by dropping the index $i$ without loss of generality, the optical channel's total Direct Current (DC) attenuation from each VLC AP to the receiver is given by

$$
H(0)= \begin{cases}\frac{(m+1) D_{\mathrm{PA}}}{2 \pi r^{2}} \cos ^{m}(\phi) T_{s}(\psi) g(\psi) \cos (\psi), & \psi \leq \psi_{\mathrm{F}}, \\ 0, & \psi>\psi_{\mathrm{F}},\end{cases}
$$

where the Lambert index $m$ depends on the semi-angle $\phi_{1 / 2}$ at half-illuminance of the source, which is given by $m=$ $-1 / \log _{2}\left(\cos \phi_{1 / 2}\right) \cdot D_{\mathrm{PA}}$ is the detector's physical area for a Photo-Diode (PD), $r$ is the distance between the VLC transmitter and the receiver, $\phi$ is the angle of irradiance, $\psi$ is the angle of incidence and $\psi_{\mathrm{F}}$ represents half of the receiver's Field-OfView (FOV). In a direct LOS path, the irradiant angle equals to the incident angle. Still referring to (2), $T_{s}(\psi)$ and $g(\psi)$ denote the gain of the optical filter and of the optical concentrator employed, respectively, while $g(\psi)$ can be written as [3]

$$
g(\psi)= \begin{cases}\frac{n^{2}}{\sin ^{2} \psi_{\mathrm{F}}}, & 0 \leq \psi \leq \psi_{\mathrm{F}} \\ 0, & \psi>\psi_{\mathrm{F}}\end{cases}
$$

where $n$ is the refractive index of a lens at a PD. Our parameter values are summarized in Table I.

According to [6], the average received power including all reflections may be negligible compared with the direct received average power of the LOS path. Therefore we may ignore the reflected optical power for simplicity and consider only the LOS-power as the desired received power. As a result, when the incoming optical radiation having an average power $P_{r}$ impinging on a photodetector, the electronic current generated by the photodetector is given by

$$
\left\langle i_{\mathrm{PD}}\right\rangle=\gamma \cdot P_{r},
$$

where $\gamma[\mathrm{A} / \mathrm{W}]$ denotes the photodetector's responsivity. Let us now define the Signal to Interference plus Noise Ratio (SINR) as the aggregate electronic power received from signal set $\boldsymbol{S}_{S} \subseteq \boldsymbol{S}$ over the noise power in a bandwidth of $B$ [MHz] [5] plus the sum of the electronic power received from other optical sources in interference set $\boldsymbol{S}_{I}$, which is the complementary set of $\boldsymbol{S}_{S}$. Since the corresponding electronic power is proportional 
to the square of the amplitude of the electronic current, we can write the SINR as

$$
\xi=\frac{\gamma^{2} \sum_{i \in \boldsymbol{S}_{S}} P_{r, i}^{2}}{N_{0} B+\gamma^{2} \sum_{i \in S_{I}} P_{r, i}^{2}}
$$

where $N_{0}\left[\mathrm{~A}^{2} / \mathrm{Hz}\right]$ is the noise power spectral density dominated by the shot noise $N_{\text {shot }}$ [6], given as $N_{0} \cong N_{\text {shot }}=$ $q I_{a}\left(P_{r}\right) \sim 10^{-22}$, where $q$ denotes the electron charge and $I_{a}\left(P_{r}\right)$ is the photo-current at the receiver [5], which relies on the received power. The expression in (5) is in its common form and it will be different for each of the VLC cell formations, which are discussed next.

\section{B. Regular Cell Formation}

1) Unity Frequency Reuse: The most straightforward way of constructing a VLC cell is to simply assume that each VLC AP illuminates an individual cell, which corresponds to adopting Unity Frequency Reuse (UFR) across all cells. Fig. 1(a) shows the UFR design, where each single VLC AP illuminates an individual cell and the same frequency $f$ is reused across all cells. The shaded areas represent the Inter-Cell Interference (ICI) imposed by the LOS ray conveying different information and arriving from the neighbouring cells at the cell edge. For the triangular point shown in Fig. 1(a), the SINR is given by

$$
\xi_{\mathrm{UFR}}=\frac{\gamma^{2} P_{r, \mathrm{~A}}^{2}}{N_{0} B+\gamma^{2}\left(P_{r, \mathrm{~B}}^{2}+P_{r, \mathrm{C}}^{2}+P_{r, \mathrm{D}}^{2}\right)},
$$

which shows that ICI arrives from all the other three neighbouring cells in this scenario. If the FOV was sufficiently narrow, the ICI may be mitigated since the user can only receive data from a single VLC AP. However, this would potentially lead to isolated "coverage islands" and "coverage holes," which consequently may result into frequent horizontal handovers and outage event, when the DL user is walking between VLC APs, since the user will experience dramatic performance degradation in the area without LOS coverage.

2) Non-Unity Frequency Reuse: Following the traditional cellular design principle, non-unity Frequency Reuse (FR) patterns may be employed for reducing the ICI, while each VLC AP still functions as an individual cell. Since the first-tier neighbouring cells contribute most of the ICI, while the secondtier cells generally have a negligible influence, an FR factor of two may be used. For the triangular point shown in Fig. 1(b), the SINR is given by

$$
\xi_{\mathrm{FR}-2}=\frac{\gamma^{2} P_{r, \mathrm{~A}}^{2}}{N_{0} B / 2+\gamma^{2} P_{r, \mathrm{D}}^{2}} .
$$

Hence, the ICI emanating from the neighbouring cells $B$ and $C$ can be removed. Although this is an appealingly simple solution, when using an FR factor larger than one, the system has to obey the classic trade-off between reduced Bandwidth

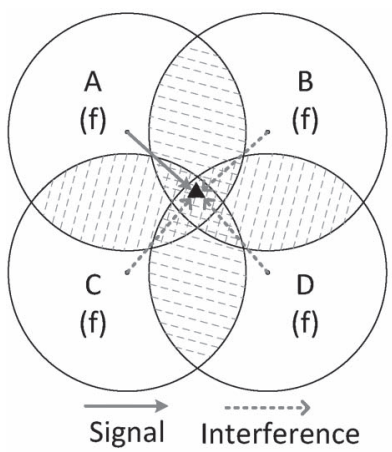

(a)

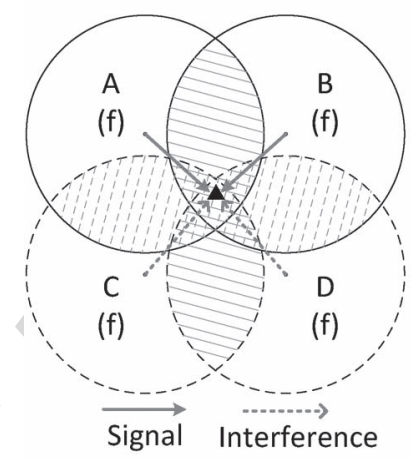

(c)

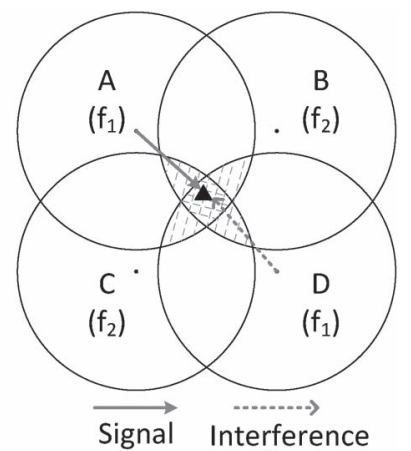

(b)

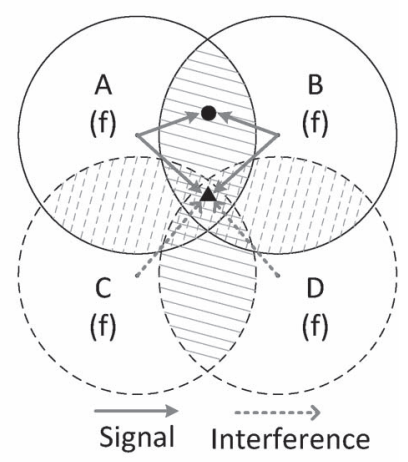

(d)
Fig. 1. Different cell formations. (a) A regular cell formation, (b) has a FR factor of two, (c) represents two merged 2-AP cells with CT, and (d) shows two merged 2-AP cells using VT. The triangle and circle denote certain points of reception. The shaded areas covered with dotted lines represent the ICI imposed by the LOS ray of neighbouring cells at the cell edge. The shaded areas covered by solid lines represent the overlapping areas within the merged 2-AP cells. In (a), (c), and (d), the entire frequency band $f$ is used by each small cell, while in (b) orthogonal frequencies $f_{1}$ and $f_{2}$ are employed by neighbouring cells, where we have $f_{1}=f_{2}=f / 2$.

Efficiency (BE) and improved cell-edge SINR. In fact, supporting mobility is the most grave problem associated with nonunity FR during VLC cell formation, since switching between frequencies every few meters during the user's movement degrades the user experience. This is also the reason for not considering FFR [15], which exhibits a more elaborate frequency planning and triggers even more frequent handovers.

\section{Merged Cell Formation}

To reduce the size of the ICI-infested areas, whilst improving the mobility, several neighbouring cells can be merged into a large multi-AP cell, where advanced transmission techniques may be employed in their overlapping areas. In the following, we use UFR across multi-AP cells for simplicity, although nonunity FR might be also used.

1) Combined Transmission: In this arrangement, each individual VLC AP of a multi-AP cell conveys the same information on the same visible carrier frequency in their overlapping areas. In Fig. 1(c), $A$ and $B$ are merged into a 2-AP cell and transmit identical signals in their overlapping area as a single source, which we refer to as Combined Transmission (CT). Thus the potential ICI is beneficially turned into useful signals which may be combined and the original cell edges of Fig. 1(a) 
become the cell centers of Fig. 1(c). For the triangular point shown in Fig. 1(c), the SINR is given by

$$
\xi_{\mathrm{CT}-2}=\frac{\gamma^{2}\left(P_{r, \mathrm{~A}}^{2}+P_{r, \mathrm{~B}}^{2}\right)}{N_{0} B+\gamma^{2}\left(P_{r, \mathrm{C}}^{2}+P_{r, \mathrm{D}}^{2}\right)} .
$$

Although the SINR may be enhanced, CT results in a reduced $\mathrm{BE}$, since only a single user is served at a time by several APs in the overlapping area within a merged cell.

2) Vectored Transmission: To eliminate the BE-reduction imposed by CT, Zero-Forcing (ZF)-based Vectored Transmission (VT) techniques can be employed for serving multiple users at the same time in the overlapping area. The underlying principle of ZF-based VT is to totally eliminate the ICI at the multiple transmitters, so that the multiple users receive mutually interference-free signals.

To elaborate a little further, let $n_{\alpha}$ denote the number of APs in a merged $n_{\alpha}$-AP cell and a vector of $U$ users are served simultaneously within the overlapping area. Let further $\boldsymbol{x}_{\boldsymbol{t}} \in \mathbb{R}^{U \times 1}$ and $\boldsymbol{y}_{\boldsymbol{r}} \in \mathbb{R}^{U \times 1}$ denote the vectors of transmitted and received signals, respectively. By using VT, we have

$$
\boldsymbol{y}_{\boldsymbol{r}}=\gamma P_{t} \boldsymbol{H} \boldsymbol{G} \boldsymbol{\Omega} \boldsymbol{x}_{\boldsymbol{t}}+\boldsymbol{n},
$$

where $\boldsymbol{n}$ includes both the noise and the ICI received from the neighbouring merged cells, while the channel-matrix $\boldsymbol{H} \in$ $\mathbb{R}^{U \times n_{\alpha}}$ hosts the DC attenuations between $U$ users and $n_{\alpha}$ APs. Furthermore, the matrix $\boldsymbol{G}=\boldsymbol{H}^{H}\left(\boldsymbol{H} \boldsymbol{H}^{H}\right)^{-1}$ obeys the ZF criterion, which hence results in a beneficial ICI-free identity matrix for $\boldsymbol{H} \boldsymbol{G}=\boldsymbol{I}_{U}$. Finally, the matrix $\boldsymbol{\Omega}$ is introduced to enforce the per-AP power constraints. According to [28], we have

$$
\boldsymbol{\Omega}=\omega \boldsymbol{I}_{U}, \quad \omega=\min _{\alpha=1,2, \ldots, n_{\alpha}} \sqrt{\frac{1}{\|\boldsymbol{G}(\alpha,:)\|_{F}^{2}}},
$$

where $\boldsymbol{G}(\alpha,:)$ is the $\alpha$ th row of $\boldsymbol{G}$. As a result, the SINR of a particular user may be written as ${ }^{3}$

$$
\xi_{\mathrm{JT}}=\frac{\gamma^{2} P_{t}^{2} \omega^{2}}{N_{0} B+\gamma^{2} \sum_{i \in \boldsymbol{S}_{I}} P_{r, i}^{2}} .
$$

In general, to facilitate VT from $n_{\alpha}$ APs to $U$ users, both the $\left(U \times n_{\alpha}\right)$-element DC attenuation matrix and the users' data have to be shared amongst the $n_{\alpha}$ APs [29]. For VLC channels, the requirements may be readily satisfied, since the VLC users are predominantly stationary. This is similar to the successful employment of ZF-based Transmit Pre-Coding (TPC) techniques, which is referred to as VT in the stateof-the-art Digital Subscriber Line (DSL) based G.fast system (Recommendation ITU-T G.9701) invoked for coping with the Far End Cross Talk (FEXT) between twisted pairs.

\footnotetext{
${ }^{3}$ Since the interference power received by the merged cell under consideration is influenced by the ZF-based VT within its neighbouring merged cells, for simplicity, we assume that the interference imposed is always equal to its maximum value, which characterizes the worst-case situation in our VT cell formation scenario.
}

As an example, in Fig. 1(d), $A$ and $B$ form a VT-aided 2-AP cell and two users are served simultaneously in the originally ICI-infested areas. For the paired points shown in Fig. 1(d), the SINRs are given by

$$
\xi_{\mathrm{JT}-2}^{\bullet}=\frac{\gamma^{2} P_{t}^{2} \omega^{2}}{N_{0} B} ; \quad \xi_{\mathrm{JT}-2}^{\boldsymbol{\Delta}}=\frac{\gamma^{2} P_{t}^{2} \omega^{2}}{N_{0} B+\gamma^{2}\left(P_{r, \mathrm{C}}^{2}+P_{r, \mathrm{D}}^{2}\right)} .
$$

\section{Area Spectral Efficiency}

To gain further quantitative insights, Fig. 2 illustrates both the classic BE surface and the Mean Bandwidth Efficiency (MBE) of different VLC cell formations. The BE is calculated as

$$
\begin{aligned}
\eta_{\mathrm{UFR}} & =\log _{2}\left(1+\xi_{\mathrm{UFR}}\right) ; \quad \eta_{\mathrm{FR}-2}=\frac{1}{2} \log _{2}\left(1+\xi_{\mathrm{FR}-2}\right) ; \\
\eta_{\mathrm{CT}} & =\hbar \log _{2}\left(1+\xi_{\mathrm{CT}-2}\right) ; \quad \eta_{\mathrm{JT}}=\log _{2}\left(1+\xi_{\mathrm{JT}}\right) ;
\end{aligned}
$$

where $\hbar$ accounts for the loss of resource under utilisation of CT, which is 0.9122 and 0.8737 when the FOV is $120^{\circ}$ and $125^{\circ}$ under our simulation setup, respectively. ${ }^{4}$ In our simulations, a $15 \mathrm{~m} \times 15 \mathrm{~m} \times 3 \mathrm{~m}$ room model is considered, including $4 \times 4$ uniformly distributed optical APs at a height of $2.5 \mathrm{~m}$. The parameters of the VLC APs are summarized in Table I. Compared to UFR as shown in Fig. 2(a), a FR of two sufficiently reduces the ICI-contaminated areas, but results into a significantly reduced $\mathrm{BE}$ as shown in Fig. 2(b). By contrast, all of our proposed merged cell formations shown in Fig. 2(c)-(e) improve the MBE, as suggested by Fig. 2(f). More explicitly, when a 2-AP cell is created, the MBE of VT is only marginally better than that of CT. However, when forming all VLC APs as a single cell, a substantial MBE improvement can be achieved with the aid of VT, since the resultant system becomes reminiscent of a large-scale Multi-Input Multi-Output (MIMO) system.

\section{Methodology}

Let us now determine the optimum LB for a set of users in this hybrid VLC/WiFi system by taking into account various VLC cell formations. We would like to introduce some notations first. Let $\boldsymbol{C}$ be the set of the single-AP or multi-AP VLC cells, where $|\boldsymbol{C}|=C$ is the number of cells. In this section, we illustrate our methodology in the UFR scenario, which can be readily extended to other cell formations. $W$ denotes the set of the WiFi APs and we have $|\boldsymbol{W}|=1$. At the same time, the users of the set $\boldsymbol{U}$ are assumed to be uniformly distributed at random in this hybrid VLC/WiFi system. Since each user has a limited FOV, they can only communicate with each other using VLC, if one or more optical APs reside within the FOV of the user. Let $\boldsymbol{R}^{\mathrm{VLC}}$ be the matrix of throughput between the VLC cells $\alpha$ and the user $\mu$, which is defined as $\boldsymbol{R}^{\mathrm{VLC}}=\left(r_{\alpha \mu}: \alpha \in\right.$

${ }^{4}$ In Fig. 2(c), $\hbar$ is given by

$$
\hbar=\frac{1}{2} \cdot \frac{D_{s}}{D_{m c}}+\frac{D_{m c}-D_{s}}{D_{m c}}
$$

where $D_{s}$ and $D_{m c}$ denote the area of the shaded areas within the merged 2 -AP cell and the total area of the merged cell, respectively. 


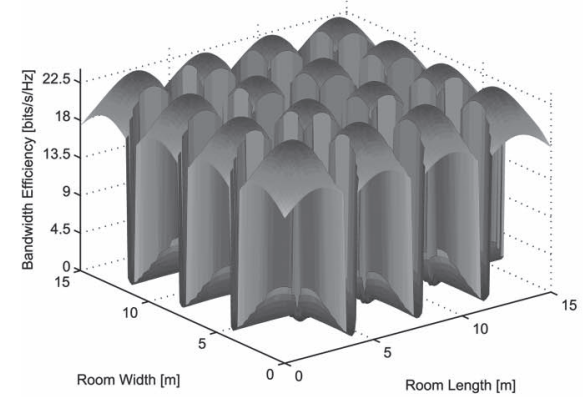

(a) UFR

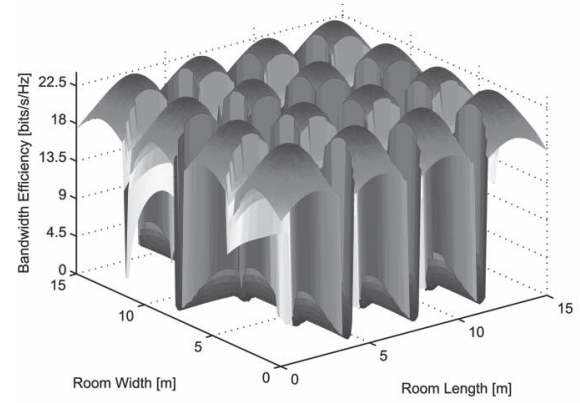

(d) VT-2

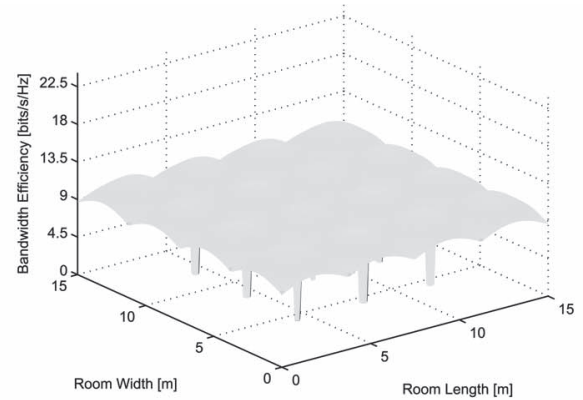

(b) FR-2

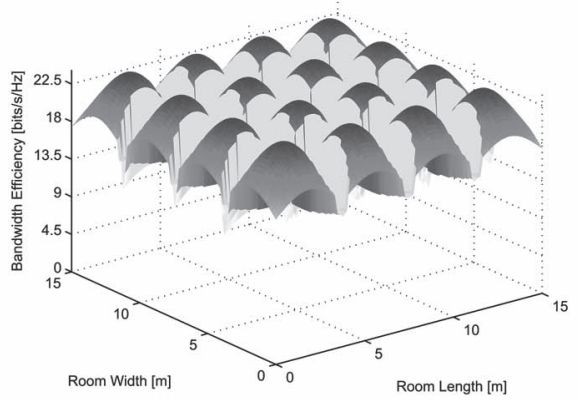

(e) VT-16

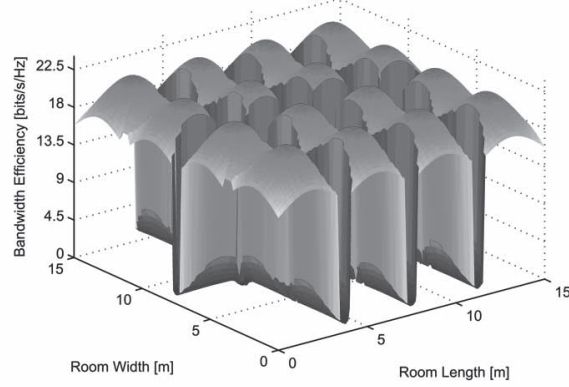

(c) $\mathrm{CT}-2$

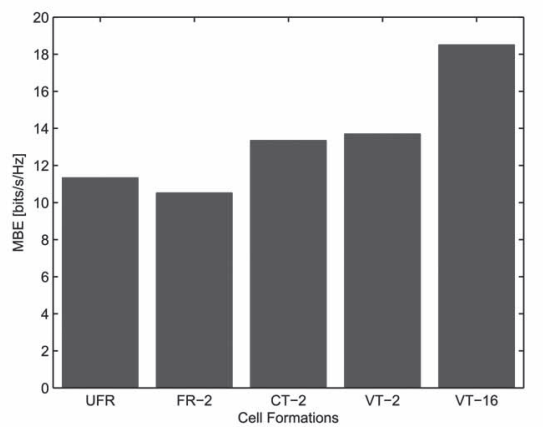

(f) MBE

Fig. 2. BE surface and the MBE of different VLC cell formations. (a) A regular UFR cell formation, (b) has a FR factor of two, (c) represents merged 2-AP cells with CT, (d) shows merged 2-AP cells with VT, (e) is a merged 16-AP cell with VT, and (f) shows the MBE of (a)-(e), where the receivers' FOV is $120^{\circ}$.

$\boldsymbol{C}, \boldsymbol{\mu} \in \boldsymbol{U})$. Let furthermore $\boldsymbol{R}^{\mathrm{WiFi}}=\left(r_{\alpha \mu}: \alpha \in \boldsymbol{W}, \mu \in \boldsymbol{U}\right)$ be the matrix of throughput allocated by the WiFi AP to the user $\mu$.

The LB problem is a connection level issue, which can be performed according to the connection level statistics [20]. Since no fast-fading is experienced in VLC propagation, as long as the users' positions remain static, the channel information does not change. When considering a low-mobility indoor scenario, the coherence time of WiFi propagation is sufficiently long. Hence, both the VLC and WiFi propagation changes slowly in the given period. The required channel information only has to be collected infrequently before activating this optimization procedure. As a result, we consider a static system without the arrival of new users and the departure of existing users. In general, this resorts to the NUM framework [17] and three methodologies will be employed in this section. We commence with an exact non-linear formulation and then discretize this non-linear formulation. Furthermore, we come to the classic distributed algorithm, which is capable of approaching the performance of the near-optimal centralized solution.

\section{A. Centralized Approach}

Our objective is to find the optimal LB using the Objective Function (OF) of maximizing the sum of all users' utility functions under the PF constraints [20] in this hybrid VLC/WiFi system, which is ultimately a joint association control and resource allocation problem. In this context, the authors of [30] have shown that we can achieve proportional allocation by optimizing the OF of:

$$
\operatorname{maximize} \sum_{\mu \in \boldsymbol{U}} \log \left(\beta_{\mu}\right),
$$

where $\beta_{\mu}$ is the actual throughput allocated to a user $\mu$. The logarithm of $\beta_{\mu}$ may be interpreted as the utility function of a user, as argued for example in [31].

At this stage, a binary variable $x_{\alpha \mu}$ is introduced to indicate, whether the user $\mu$ is assigned to the AP $\alpha$, where we have $x_{\alpha \mu}=1$ if $\mu$ and $\alpha$ do have a connection, while 0 otherwise. Note that $\alpha$ may denote either a certain VLC cell or the WiFi $\mathrm{AP}$, namely $\alpha \in \boldsymbol{C} \cup \boldsymbol{W}$ in this section. Hence, the actual throughput $\beta_{\alpha \mu}$ of the user $\mu$ allocated by the AP $\alpha$ may be expressed as $x_{\alpha \mu} r_{\alpha \mu} p_{\alpha \mu}$, where $p_{\alpha \mu}$ is a fractional variable between 0 and 1, which is denoted as the proportion of time that $\alpha$ is allocated to $\mu$. For a given user $\mu$, the total actual throughput becomes

$$
\beta_{\mu}=\sum_{\alpha \in C \cup W} x_{\alpha \mu} r_{\alpha \mu} p_{\alpha \mu} .
$$

By substituting (15) into the OF of (14), we have

$$
\Gamma(\boldsymbol{x}, \boldsymbol{p})=\sum_{\mu \in \boldsymbol{U}} \log \sum_{\alpha \in \boldsymbol{C} \cup \boldsymbol{W}} x_{\alpha \mu} r_{\alpha \mu} p_{\alpha \mu} .
$$

Since only a single AP $\alpha^{\prime}$ is assigned to a given user, i.e., we have $x_{\alpha^{\prime} \mu}=1$, we can write: $\log \sum_{\alpha} x_{\alpha \mu} r_{\alpha \mu} p_{\alpha \mu}=$ $\sum_{\alpha} x_{\alpha \mu} \log \left(r_{\alpha \mu} p_{\alpha \mu}\right)$. Therefore (16) may equivalently be written as

$$
\Gamma(\boldsymbol{x}, \boldsymbol{p})=\sum_{\mu \in \boldsymbol{U}} \sum_{\alpha \in \boldsymbol{C} \cup \boldsymbol{W}} x_{\alpha \mu} \log \left(r_{\alpha \mu} p_{\alpha \mu}\right) .
$$

Several optimization constraints may be formulated based upon various assumptions as well as on a range of practical 
limitations. The mathematical formulation of this maximization problem becomes:

$$
\begin{array}{ll}
\text { maximize } & \Gamma(\boldsymbol{x}, \boldsymbol{p}) \\
\text { subject to } & \sum_{\alpha \in \boldsymbol{C} \cup \boldsymbol{W}} x_{\alpha \mu}=1 \quad \forall \mu \in \boldsymbol{U} ; \\
& \sum_{\mu \in \boldsymbol{U}} x_{\alpha \mu} p_{\alpha \mu} \leq 1 \quad \forall \alpha \in \boldsymbol{C} ; \\
& \sum_{\mu \in \boldsymbol{U}} x_{\alpha \mu} p_{\alpha \mu} \leq p_{\mathrm{DL}} \quad \forall \alpha \in \boldsymbol{W} ; \\
& x_{\alpha \mu} \in\{0,1\} \quad \forall \alpha \in \boldsymbol{C} \cup \boldsymbol{W}, \forall \mu \in \boldsymbol{U} ; \\
& 0 \leq p_{\alpha \mu} \leq 1 \quad \forall \alpha \in \boldsymbol{C} \cup \boldsymbol{W}, \forall \mu \in \boldsymbol{U} .
\end{array}
$$

Firstly, for each user $\mu$, there is one and only one AP $\alpha^{\prime}$ to satisfy $x_{\alpha^{\prime} \mu}=1$ for an extended period of time. At this stage, we do not impose any constraint on the users' resource demand, hence all the users may either connect to the VLC APs or to the WiFi AP. Secondly, each AP has to request resources confined to its maximum capacity. By contrast, for the DL WiFi AP, the available normalized capacity is assumed to be less than $p_{\mathrm{DL}}$ instead of 1 , because it may allocate $\left(1-p_{\mathrm{DL}}\right)$ of its total resources for example to the uplink. Furthermore, $p_{\mathrm{DL}}$ may be set up as any feasible value between 0 and 1 according to the specific system design and traffic requirements. Finally, the variable $x_{\alpha \mu}$ should be binary, while $p_{\alpha \mu}$ is a real value between 0 and 1. As a result, the above problem represents an MixedInteger Non-Linear Programming (MINLP) problem that may be solved directly by using the OPTI Toolbox, albeit this would be time-consuming.

\section{B. Discretized Linear Programming Approximation}

Instead of solving the MINLP directly, we might opt for a linear relaxation of the original problem. Following the work in [20], we discretize the scheduling time period of each access point into $T$ discrete intervals, where $T$ is $\kappa$ times the number of the users. In practice, $\kappa=10$ turns out to be sufficient to obtain an acceptable approximation of the non-linear problem. A new binary variable $y_{\alpha \mu t}$ is introduced. If and only if the access point $\alpha$ is associated with the user $\mu$ and allocates $t(0 \leq$ $t \leq T)$ time slots to user $\mu$, we arrive at $y_{\alpha \mu t}=1$. Thus, we arrive at the linear OF of

$$
\Gamma_{D}(\boldsymbol{y})=\sum_{\mu \in \boldsymbol{U}} \sum_{\alpha \in \boldsymbol{C} \cup \boldsymbol{W}} \sum_{t=1}^{T} y_{\alpha \mu t} \cdot \log \left(r_{\alpha \mu} \frac{t}{T}\right) .
$$

Accordingly, our linear program is then formulated as

$$
\begin{array}{ll}
\text { maximize } & \Gamma_{D}(\boldsymbol{y}) ; \\
\text { subject to } & \sum_{\alpha \in \boldsymbol{C} \cup \boldsymbol{W}} \sum_{t=1}^{T} y_{\alpha \mu t}=1 \quad \forall \mu \in \boldsymbol{U} ; \\
& \sum_{\mu \in \boldsymbol{U}} \sum_{t=1}^{T} y_{\alpha \mu t} \cdot \frac{t}{T} \leq 1 \quad \forall \alpha \in \boldsymbol{C} ; \\
& \sum_{\mu \in \boldsymbol{U}} \sum_{t=1}^{T} y_{\alpha \mu t} \cdot \frac{t}{T} \leq p_{\mathrm{DL}} \quad \forall \alpha \in \boldsymbol{W} ; \\
y_{\alpha \mu t} \in\{0,1\} \quad \forall \alpha \in \boldsymbol{C} \cup \boldsymbol{W}, \forall \mu \in \boldsymbol{U} .
\end{array}
$$

The first constraint of (20) states that only one and exactly one AP is assigned to each user. The second and third indicate that each AP restricts its time-resource allocation to capacity. This discretized linear problem has been solved with the aid of the CPLEX solver. Then we translate the solution in (20) to the non-linear program (18) as follows:

$$
x_{\alpha \mu}=\sum_{t=1}^{T} y_{\alpha \mu t}, \quad p_{\alpha \mu}=\sum_{t=1}^{T} \frac{t}{T} y_{\alpha \mu t} .
$$

\section{A Dual Decomposition Method}

We now conceive an efficient and scalable distributed algorithm for (18).

1) Transformation: According to [20], when the AP/user association matrix $\boldsymbol{x}$ is given, the unique optimal solution becomes $p_{\alpha \mu}=x_{\alpha \mu} / N_{\alpha}$, where $N_{\alpha}=\sum_{\mu \in \boldsymbol{U}} x_{\alpha \mu}$ is the number of users associated with the access point $\alpha$. As a result, the optimization in (18) may be transformed to a pure association control problem, hence we now have the primal OF of:

$$
\Gamma_{d e}(\boldsymbol{x}, \mathcal{N})=\sum_{\mu \in \boldsymbol{U}} \sum_{\alpha \in \boldsymbol{C} \cup \boldsymbol{W}} x_{\alpha \mu} \log \left(\frac{r_{\alpha \mu}}{N_{\alpha}}\right) .
$$

Additionally, we have three constraints as well:

$$
\begin{aligned}
& \sum_{\mu \in \boldsymbol{U}} x_{\alpha \mu}=N_{\alpha} \quad \forall \alpha \in \boldsymbol{C} \cup \boldsymbol{W} ; \\
& \sum_{\alpha \in \boldsymbol{C} \cup \boldsymbol{W}} x_{\alpha \mu}=1 \quad \forall \mu \in \boldsymbol{U} ; \\
& x_{\alpha \mu} \in\{0,1\} \quad \forall \alpha \in \boldsymbol{C} \cup \boldsymbol{W}, \forall \mu \in \boldsymbol{U} .
\end{aligned}
$$

Furthermore, the dual objective $g(\boldsymbol{\nu})$ is formulated as

$$
g(\boldsymbol{\nu})=\sup _{\boldsymbol{x}, \mathcal{N}} L(\boldsymbol{x}, \mathcal{N}, \boldsymbol{\nu})
$$

where $L(\boldsymbol{x}, \mathcal{N}, \boldsymbol{\nu})$ is the Lagrangian function for (22) using the constraints of (23) after relaxation of (25), yielding

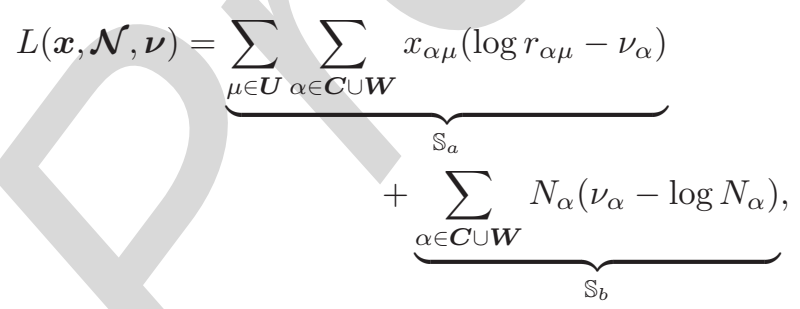

where $\mathcal{N}=\left(N_{\alpha}: \alpha \in \boldsymbol{C} \cup \boldsymbol{W}\right)$ constitutes a set, whose elements are given by the number of users associated with each AP, while $\boldsymbol{\nu}=\left(\nu_{\alpha}: \alpha \in \boldsymbol{C} \cup \boldsymbol{W}\right)$ is the vector of Lagrangian multipliers corresponding to the constraint of (23).

2) Decomposition: The problem of (26) is further partitioned into two sub-problems in a distributive-rather than centralized-fashion.

- On the user's side, we solve the sub-problem of maximizing $\mathbb{S}_{a}$. Since we have $x_{\alpha \mu} \in\{0,1\}$ and $\sum_{\alpha \in \boldsymbol{C} \cup \boldsymbol{W}} x_{\alpha \mu}=1$, for each $\mu \in \boldsymbol{U}$ we find

$$
\alpha^{*}=\underset{\alpha \in \boldsymbol{C} \cup \boldsymbol{W}}{\arg \max }\left(\log r_{\alpha \mu}-\nu_{\alpha}\right) .
$$


Then we set $x_{\alpha^{*} \mu}=1$. Hence we could compute the number of users $\mathcal{N}^{(1)}$ associated with each AP corresponding to $\mathbb{S}_{a}$.

- On the AP's side, upon applying the Karush-Kuhn-Tucker (KKT) conditions [32], for each $\alpha \in \boldsymbol{C} \cup \boldsymbol{W}$ we have

$$
\frac{\partial g^{(2)}}{\partial N_{\alpha}}=\nu_{\alpha}-\log N_{\alpha}+1=0 \Rightarrow N_{\alpha}=\exp \left(\nu_{\alpha}-1\right)
$$

Then we can obtain $\mathcal{N}^{(\mathbf{2})}$ corresponding to $\mathbb{S}_{b}$.

A variable $\delta$ is introduced to reflect the difference between the resource demand $\mathbb{S}_{a}$ and resource supply $\mathbb{S}_{b}$, defined as

$$
\delta=\left|\mathcal{N}^{(1)}-\mathcal{N}^{(2)}\right| .
$$

An acceptable target difference of $\delta_{\text {target }}$ is pre-set before iteratively solving the sub-problems. If each element of $\delta$ is smaller than the target gap, the iterations will be curtailed and the allocation result of $(\hat{\boldsymbol{x}}, \hat{\mathcal{N}})$ is obtained; otherwise $\boldsymbol{\nu}$ will be adjusted for the next iteration according to the gradient method, which is given as

$$
\nu_{\alpha}(i+1)=\nu_{\alpha}(i)-\varepsilon(i)\left(N_{\alpha}-\sum_{\mu \in \boldsymbol{U}} x_{\alpha \mu}\right),
$$

where $\varepsilon(i)$ is a sufficiently small step size in the $i$ th iteration. In this paper, we $\operatorname{set} \varepsilon(i)=\varepsilon_{0} \cdot i^{-\frac{1}{2}+\tau}$, where $\varepsilon_{0}$ and $\tau$ are positive constants appropriately chosen for satisfying $\lim _{i \rightarrow \infty} \varepsilon(i)=$ 0 and $\sum_{i=0}^{i=\infty} \varepsilon(i)=\infty$ for achieving the convergence of the gradient algorithm towards the optimal solution.

\section{Optimality Analysis}

Although the convergence of the distributed algorithm may be proved analytically, e.g. in [25], it is difficult to theoretically study how far the converged solution found is away from that of the true original problem, since the original problem contains a binary integer. Upon comparing the solutions provided by LP approximation, we experimentally study the optimality of the solution provided by the distributed algorithm, which has not been discussed in the literature.

1) Justification: Let us now consider the relationship between the OF value $\Gamma$ of the original MINLP problem (18), the LP OF value $\Gamma_{D}$ for (20) and the solution $\hat{\Gamma}_{d e}$ provided by the distributed algorithm. Since the distributed algorithm is performed after relaxing (25), which prevents us from theoretically proving the optimality of the solution $\hat{\Gamma}_{d e}$ of the problem (22), we opt for an empirical analysis of the optimality of $\Gamma$, $\Gamma_{D}$ and $\hat{\Gamma}_{d e}$.

(i) NLP vs. LP: The authors of [20] have characterized the relationship between the NLP and LP formulations for any feasible solution $(\boldsymbol{y})$ and its corresponding translation $(\boldsymbol{x}, \boldsymbol{p})$, which may be written as

$$
\Gamma_{D} \leq \Gamma \text {. }
$$

(ii) NLP vs. Distributed: We would like to introduce the notation (23') for the relaxation of the problem of (22).

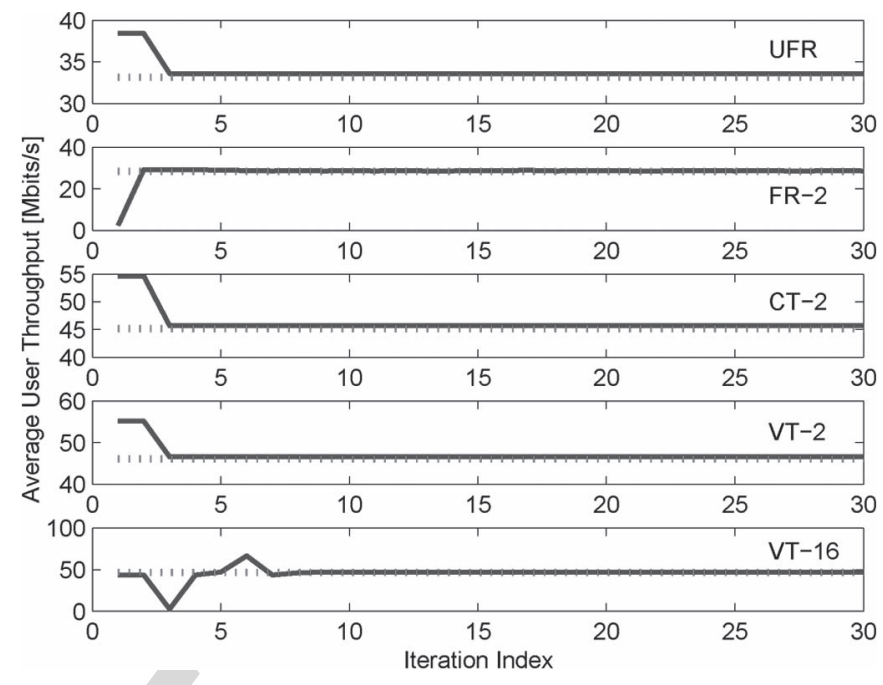

Fig. 3. Empirical optimality of the distributed algorithm relying on the simulation parameters discussed in Section IV-A. The solid line shows the average user throughput in each iteration, while small vertical tick-marks show the solution of the LP problem provided by CPLEX.

Furthermore, we know that $\hat{\Gamma}_{d e}$ upper bounds (23') [32]. Intuitively, $\hat{\Gamma}_{d e}$ may also upper bound (22), since the solution of $x_{\alpha \mu}$ always satisfies the constraint (25) in the process of solving (23').

(iii) NLP, LP and Distributed: Combing (i) and (ii) above, for any feasible pair, we may use $\hat{\Gamma}_{d e}$ and $\Gamma_{D}$ as the upper bound and the lower bound for the original MINLP problem, respectively. Please note that all the above discussions are here related to the resource allocation lemma detailed at the beginning of Section III-C [20]. Under this lemma (18) may be written as (22).

Fig. 3 shows the empirical optimality of our distributed algorithm with the aid of simulations relying on the parameters discussed in Section IV-A. The discretized LP approximation is implemented in our simulation scenarios and the near-optimal solution of the LP OF is shown by the dashed line in Fig. 3. We obtain a fairly accurate solution of the distributed OF within a dozen of iterations, which is within a margin of about $1.5 \%$ from the sub-optimal LP solution. Since we may derive from (iii) that the optimal NLP OF value $\Gamma$ may be between $\Gamma_{D}$ and $\hat{\Gamma}_{d e}$, the difference between $\hat{\Gamma}_{d e}$ and $\Gamma$ may be even smaller. This demonstrates that the distributed algorithm is capable of converging to the optimal value of the original NLP problem, whilst providing a near-optimal solution within a few dozens of iterations.

2) Implementation: As a benefit of the above optimality analysis, we opt for the distributed algorithm. Indeed, the distributed algorithm converges to a near-optimal solution significantly faster than the centralized approaches. In each iteration, each AP initializes and broadcasts a feasible price value $\nu_{\alpha}$ to all the users within its coverage. Here we assume that different APs use pre-allocated orthogonal frequency bands for simultaneously broadcasting $\nu_{\alpha}$. Each user finds the optimal AP $\alpha^{*}$ according to (28) and sends its user-identifier back to $\alpha^{*}$. Thus each AP becomes capable of calculating the user demand $\mathcal{N}^{(1)}$. At the same time, the supply vector $\mathcal{N}^{(2)}$ can also be calculated by the APs. Each AP compares its supply and user 
demand. When the difference between the demand and supply becomes sufficiently small, the iterations are curtailed and a near-optimal solution has been found. In this way, each AP becomes capable of performing its own resource allocation. The total number of information exchange operations is proportional to $\left(n_{\alpha}+n_{\mu}\right)$, where $n_{\alpha}$ and $n_{\mu}$ denote the number of APs and users, respectively. Let us now provide an overview of the dual decomposition algorithm in form of Algorithm 1, which has been verified using our simulations.

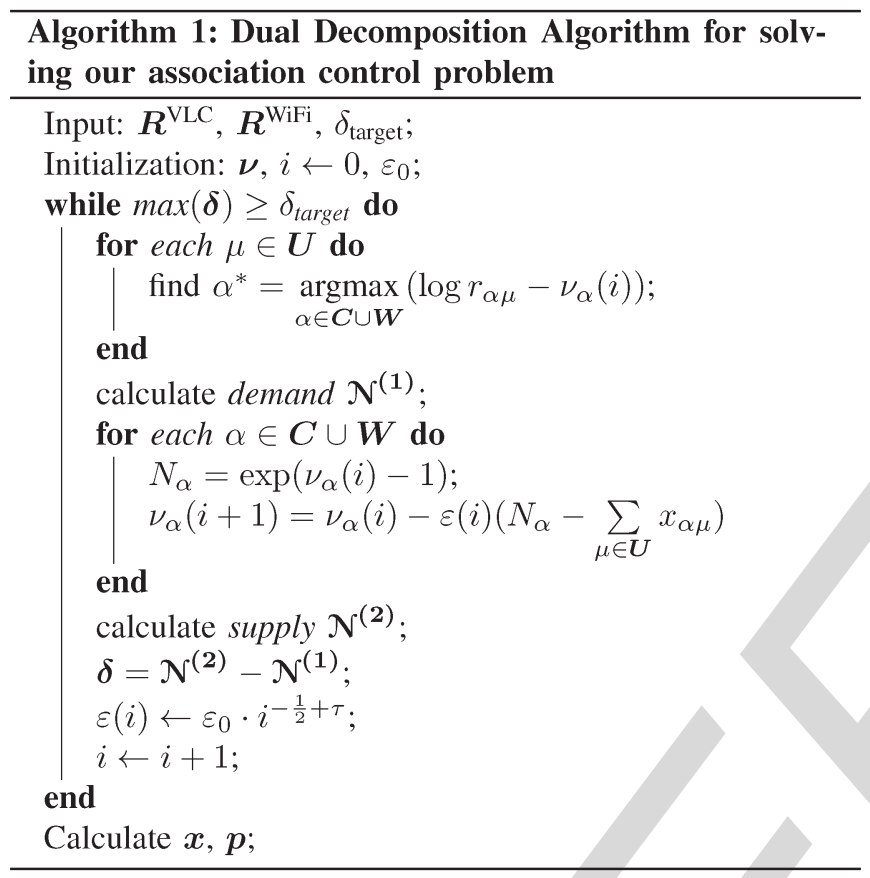

\section{Performance Evaluations}

In this section, we will present our simulation results for the LB problem, with special emphasis on the aforementioned various VLC cell formations in our hybrid VLC/WiFi system. We commence by studying the user's average throughput for different FOV and LOS blocking probability, followed by investigating the fairness both from the system's and the individual users' perspectives for the sake of characterizing the quality of service experienced by the users under different cell formation schemes.

\section{A. Simulation Setup}

A $15 \mathrm{~m} \times 15 \mathrm{~m} \times 3 \mathrm{~m}$ room model is considered, which is only covered by a VLC system including $4 \times 4$ uniformly distributed optical APs at a height of $2.5 \mathrm{~m}$. Additionally, the room is entirely covered by an IEEE Std 802.11n WiFi AP supporting a data rate as high as 120 Mbits/s within $25 \mathrm{~m}$. The parameters of the LED lamps are summarized in Table I. The normalized WiFi DL capacity $p_{\mathrm{DL}}$ is assumed to be 0.8 and $U=50$ users are assumed to be distributed uniformly at random during each simulation run. All of the results shown are averaged over 50 simulation runs. To be more realistic, we introduce specific modulation schemes for calculating the users' achievable throughput. For simplicity, we consider base-

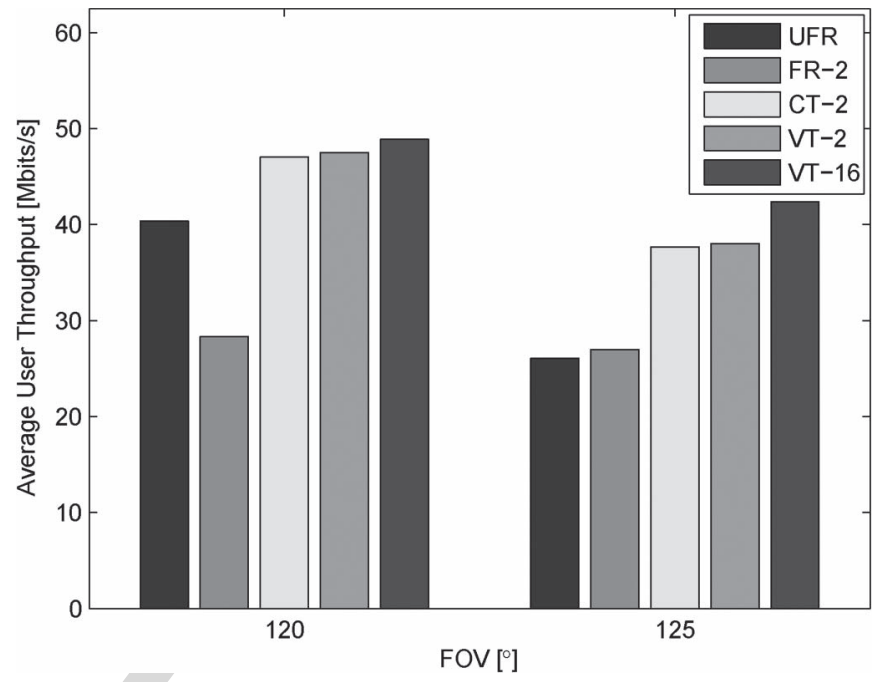

Fig. 4. Average user throughput of various cell formations with different FOV after performing LB in the hybrid VLC/WiFi system.

band transmissions without subcarrier modulation at this stage. Pulse-Amplitude Modulation having an order of $M$ ( $M$-PAM) is used. Based on our Bit Error Rate (BER) performance results, given a certain target BER, Signal to Noise Ratio (SNR) and Signal to Interference Ratio (SIR), the maximum affordable $M$-PAM order capable of maintaining the target-BER can be determined. ${ }^{5}$ Thus the attainable throughput becomes $r_{\alpha \mu}=$ $2 B \log _{2} M /(1+\rho)$, where the roll-off factor of the raisedcosine pulses is assumed to be $\rho=1$.

\section{B. Throughput Investigations}

1) Throughput Investigations for Different FOV: The FOV is one of the factors that is expected to significantly influence the ICI in VLC-based networks. Increasing the FOV leads to the expansion of the ICI-contaminated areas and correspondingly the employment of ICI reduction techniques may become more important. Fig. 4 shows the average user throughput of different cell formations with different FOV after performing LB in the hybrid VLC/WiFi system. We can see that the throughput provided by the merged cells is higher than the regular cell formations for both FOV values. Since the increase of FOV may result in 1) an increased probability of having user in the ICIcontaminated areas and 2) an decease of received signal power and an increase of received noise power, the average throughput is reduced in all cell formation scenarios for FOV $=120^{\circ}, 125^{\circ}$. In particular, the UFR scheme is the most badly affected one upon increasing the FOV, which supports the lowest throughput, when the FOV is increased to $125^{\circ}$. Furthermore, FR-2 does not change much.

\footnotetext{
${ }^{5}$ We could also consider the joint effects of noise and interference. According to [5], the relationship between the BER and $\xi$ for $M$-PAM signals is given by

$$
\mathrm{BER}_{M-\mathrm{PAM}} \cong \frac{M-1}{M} \frac{2}{\log _{2} M} Q\left(\frac{\sqrt{\xi}}{M-1}\right) .
$$

Given a certain target BER and the above-mentioned SINR value $\xi$, this is an alternative technique of determining the maximum PAM order $M$, which agrees with our simulation results.
} 

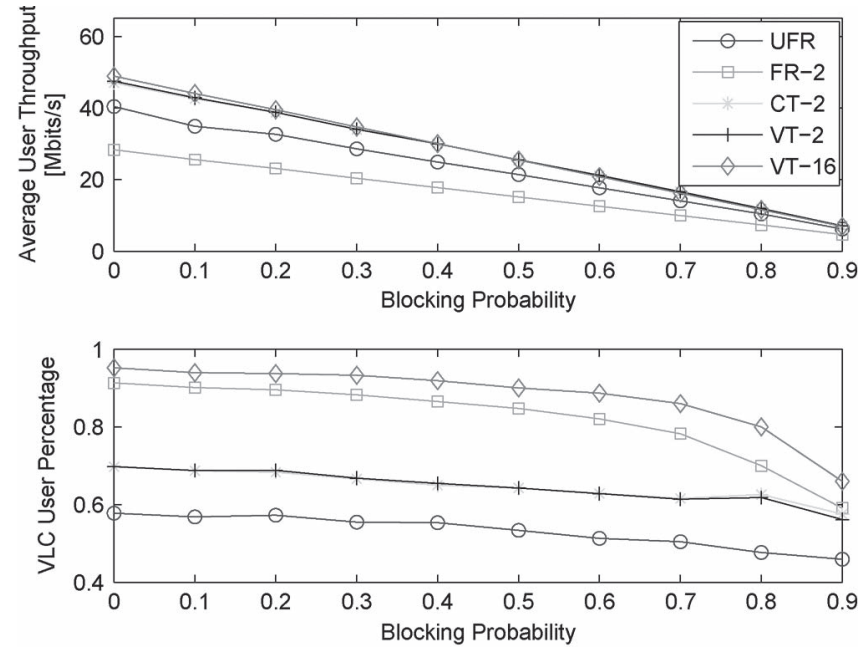

Fig. 5. Average user throughput and proportion of VLC users under various LOS blocking probabilities, $\mathrm{FOV}=120^{\circ}$.

2) LOS Blocking Analysis: As mentioned in Section I, the WiFi AP is also capable of providing seamless DL coverage, when the LOS VLC-DL receptions are blocked. We introduce the LOS blocking probability $P_{\text {block }}$ to represent the probability that the LOS VLC path is blocked, which may lead to a reduction of the data rate experienced by some VLC users. Then the VLC DL data rate becomes $\widetilde{R}=P_{\text {block }} \cdot 0+\left(1-P_{\text {block }}\right) \cdot R$. At this stage, we assume that all LOS paths are blocked with an equal probability. Fig. 5 indicates that, as expected, the average user throughput and the proportion of VLC users is reduced upon increasing the LOS blocking probability in all cell formation scenarios, hence more users are allocated to the WiFi AP. This demonstrates that the WiFi AP plays an important role in this hybrid system, especially when the LOS paths may be blocked.

\section{Fairness Grade Investigations}

1) Average Fairness: Next we will analyse both the network's average fairness as well as the individual user's fairness, given a certain total throughput. The Grade of Fairness (GOF) for the network is defined as

$$
\sigma_{a-\mathrm{VLC}}=\left|1-\frac{\mathrm{VLC}-\text { fractionof total throughput }}{\text { fraction of VLC }- \text { users }}\right|
$$

For example, in the UFR scenario, the VLC throughput proportion of the total VLC/WiFi throughput is $95.25 \%$, while $57.76 \%$ of the users is supported by the VLC APs, rather than by WiFi. Hence the grade of the average fairness becomes $\sigma_{a}=\mid 1-$ $95.25 \% / 57.76 \% \mid \approx 0.649$. The system provides the highest grade of average fairness, when the VLC (WiFi) throughput accounts for a certain proportion of the total throughput and at the same time, the number of the VLC (WiFi) users accounts for the same proportion of the total number of users in the hybrid system. Hence for the highest possible GOF we have $\sigma_{a}=0$. It is plausible that the system will provide a higher grade of average fairness, when $\sigma_{a}$ is closer to zero, provided that no

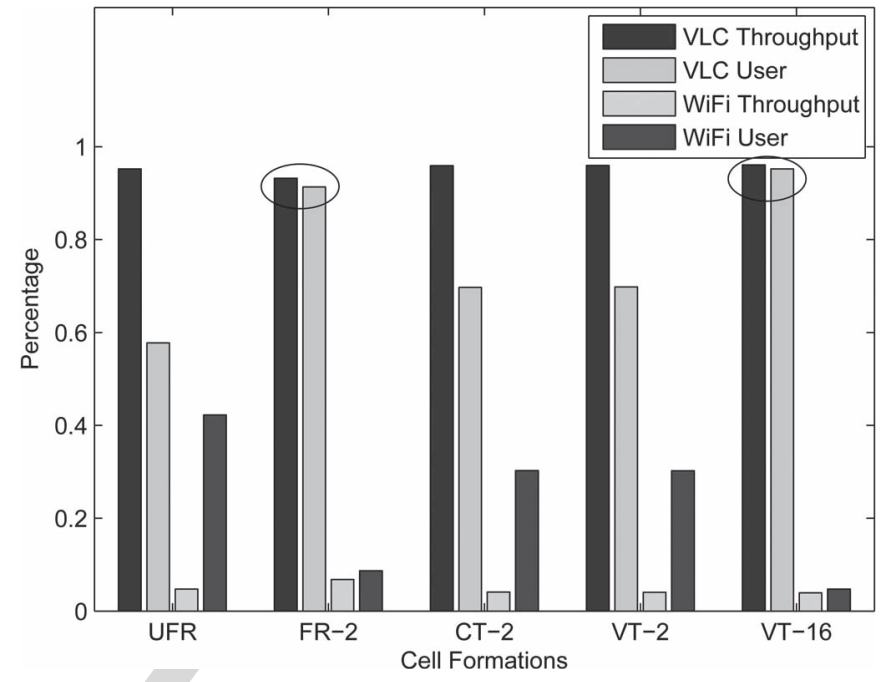

Fig. 6. Throughput and user percentage in different cell formations, FOV $=$ $120^{\circ}$

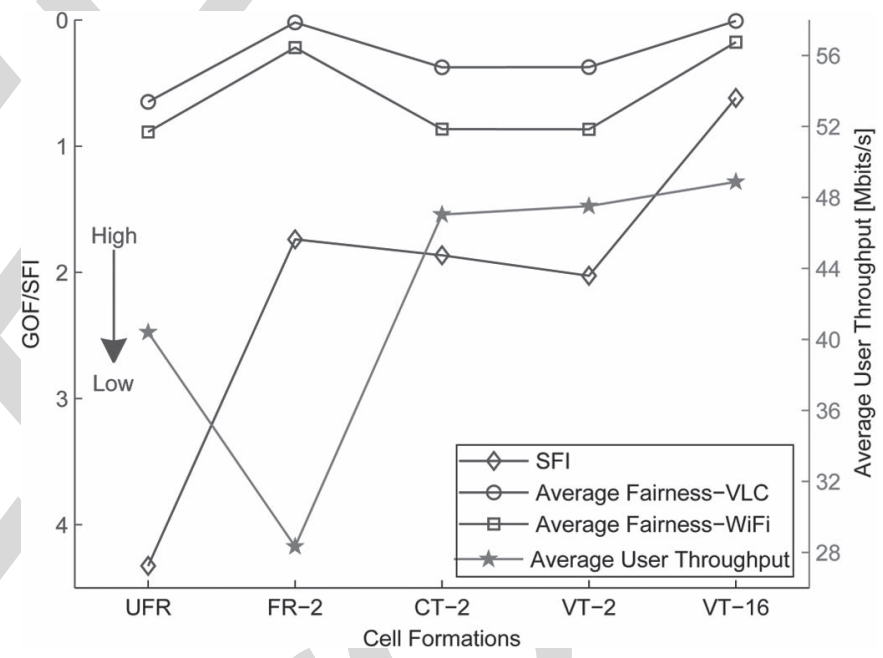

Fig. 7. The GOF, SFI and average user throughput, FOV $=120^{\circ}$. The $\mathrm{GOF} / \mathrm{SFI}$ perceived is reduced upon increasing its value.

multi-service requirements are considered at this stage [26]. Since the sum of VLC throughput (users) and WiFi throughput (users) constitutes the total throughput (users) of the system, the VLC system and the WiFi system provide a similar grade of average fairness. When the difference between the VLC (WiFi) throughput proportion and the fraction of VLC (WiFi) users is smaller, $\sigma_{a}$ is also reduced, i.e. the hybrid system provides a higher grade of average fairness.

We know that the size of ICI-contaminated areas is significantly smaller when the FR factor is two and no ICI occurs when all the VLC APs are merged into a 16-AP cell employing VT. Hence more users are expected to be supported by VLC APs in these two scenarios compared to the UFR, CT-2 and VT-2 schemes. We can see from Fig. 6 that the VLC (WiFi) network throughput and the corresponding user-proportion are closest to each other, since the FR-2 and VT-16 scenario have a higher percentage of VLC users, as indicated by the circles in Fig. 6. Specific values of $\sigma_{a}$ are plotted in Fig. 7. 


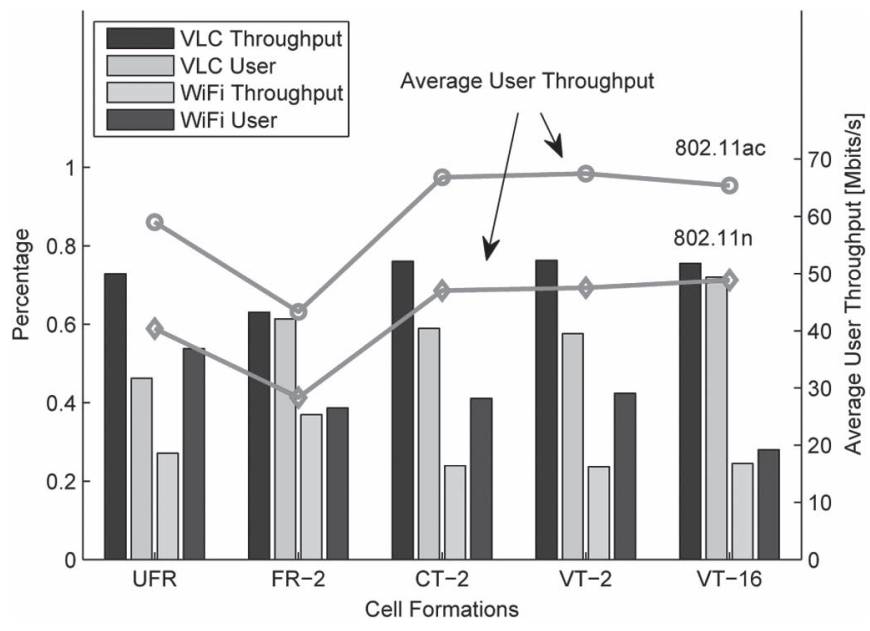

Fig. 8. Throughput and user percentage in different cell formations, FOV = $120^{\circ}$. WiFi data rate is set to $1 \mathrm{Gbits} / \mathrm{s}$.

2) Individual Fairness: We will use the Service Fairness Index (SFI) of [33] to reflect the individual fairness experienced by the users. The objective of ensuring fairness amongst the users is to guarantee that all users benefit from the same throughput within a given period, provided that the users' data rate requirements are identical. However, this is often unrealistic. The SFI of [33] is introduced to represent the difference between the maximum and the minimum amongst all the users' throughput. When the SFI is low, the throughput-difference of different users is small, hence they are served fairly. By contrast, if the SFI is high, the users experiencing a lower data rate may complain about their unfair treatment. Therefore a lower SFI means a higher grade of individual fairness. The specific SFI values of the different cell formation scenarios considered are plotted in Fig. 7. It can be clearly seen that the users' throughput-difference is the smallest in the VT-16 scheme, which is expected on the basis of Fig. 2(e), where VT-16 provides the smoothest BE distribution.

Considering the average user throughput and fairness performance comprehensively in Fig. 7, the merged 16-AP cell relying on VT is the most attractive one having the highest throughput as well as the highest GOF and SFI. However, its implementation complexity is also significantly higher than that of the regular designs and of CT.

\section{1 Gbis/s-Data-Rate WiFi}

Since 802.11ac may support a data rate over $1 \mathrm{Gbits} / \mathrm{s}$, it is realistic to investigate a hybrid system having a WiFi data rate of 1 Gbits/s. Fig. 8 shows the average user throughput, the throughput and user percentages in the different cell formations having a WiFi data rate as high as $1 \mathrm{Gbits} / \mathrm{s}$ and $\mathrm{FOV}=120^{\circ}$. The average user throughput is increased in all the scenarios considered. Both the percentage share of WiFi throughput and the proportion of its users are increased with the improvement of the WiFi data rate. The merged cells still provide a higher throughput, however the single 16-AP cell relying on VT may no longer have the absolute advantage in terms of its average throughput. This may provide some insights for this hybrid systems' design, when the WiFi data rate is increased.

\section{CONCLUSiON}

In this paper, various VLC cell formation schemes and a heterogeneous system constituted by WLANs and VLC networks were investigated. We studied the regular design concept borrowed from cellular networks relying on different FR factors in VLC environments as well as of merged multi-AP cells employing either CT or ZF-based VT. To solve the essential LB problem in the context of our VLC/WiFi hybrid system, both centralized and distributed algorithms were invoked for implementing a PF scheduler. We analysed the MBE of different VLC cell formations as well as the throughput and fairness of the hybrid VLC/WiFi system. By employing a sophisticated VT among all the 16 VLC APs, the VLC network becomes capable of providing a higher MBE, while the hybrid system is capable of providing a higher average throughput without any sacrifice of the fairness, when the WiFi data rate is modest.

\section{REFERENCES}

[1] L. Hanzo et al., "Wireless myths, realities, futures: From 3G/4G to optical and quantum wireless," in Proc. IEEE, May 2012, vol. 100, pp. 1853-1888.

[2] H. Elgala, R. Mesleh, and H. Haas, "Indoor optical wireless communication: Potential and state-of-the-art," IEEE Commun. Mag., vol. 49, no. 9, pp. 56-62, Sep. 2011.

[3] J. Kahn and J. Barry, "Wireless infrared communications," Proc. IEEE, vol. 85, no. 2, pp. 265-298, Feb. 1997.

[4] Z. Ghassemlooy, W. Popoola, and S. Rajbhandari, Optical Wireless Communications: System and Channel Modelling With MATLAB. Boca Raton, FL, USA: CRC Press, 2012.

[5] J. Grubor, S. Randel, K.-D. Langer, and J. Walewski, "Broadband information broadcasting using led-based interior lighting," J. Lightw. Technol., vol. 26, no. 24, pp. 3883-3892, Dec. 2008.

[6] T. Komine and M. Nakagawa, "Fundamental analysis for visible-light communication system using led lights," IEEE Trans. Consum. Electron., vol. 50, no. 1, pp. 100-107, Feb. 2004.

[7] S. Dissanayake and J. Armstrong, "Comparison of ACO-OFDM, DCO-OFDM and ADO-OFDM in IM/DD systems," J. Lightw. Technol., vol. 31, no. 7, pp. 1063-1072, Apr. 2013.

[8] X. Li, J. Vucic, V. Jungnickel, and J. Armstrong, "On the capacity of intensity-modulated direct-detection systems and the information rate of ACO-OFDM for indoor optical wireless applications," IEEE Trans. Commun., vol. 60, no. 3, pp. 799-809, Mar. 2012.

[9] R. Mesleh, H. Elgala, and H. Haas, "On the performance of different OFDM based optical wireless communication systems," IEEE/OSA J. Opt. Commun. Netw., vol. 3, no. 8, pp. 620-628, Aug. 2011.

[10] J. Armstrong, "OFDM for optical communications," J. Lightw. Technol., vol. 27, no. 3, pp. 189-204, Feb. 2009.

[11] H. Le-Minh et al., "100-Mb/s NRZ visible light communications using a postequalized white LED," IEEE Photon. Technol. Lett., vol. 21, no. 15, pp. 1063-1065, Aug. 2009.

[12] IEEE Standard for Local and Metropolitan Area Networks-Part 15.7: Short-Range Wireless Optical Communication Using Visible Light, IEEE Std 802.15.7-2011, Sep. 2011, pp. 1-309.

[13] M. Rahaim, A. Vegni, and T. D. C. Little, "A hybrid radio frequency and broadcast visible light communication system," in Proc. IEEE GLOBECOM Workshops, Dec. 2011, pp. 792-796.

[14] Y. Wang, S. Videv, and H. Haas, "Dynamic load balancing with handover in hybrid Li-Fi and Wi-Fi networks," in Proc. IEEE 25th Int. Symp. Pers., Indoor Mobile Radio Commun., Washington, DC, USA, 2014.

[15] C. Chen, N. Serafimovski, and H. Haas, "Fractional frequency reuse in optical wireless cellular networks," in Proc. IEEE PIMRC, Sep. 2013, pp. 3594-3598.

[16] C. Chen, D. Tsonev, and H. Haas, "Joint transmission in indoor visible light communication downlink cellular networks," in Proc. IEEE GLOBECOM Workshops, Dec. 2013, pp. 1127-1132.

[17] D. Palomar and M. Chiang, "A tutorial on decomposition methods for network utility maximization," IEEE J. Sel. Areas Commun., vol. 24, no. 8, pp. 1439-1451, Aug. 2006. 
[18] J. Andrews, S. Singh, Q. Ye, X. Lin, and H. Dhillon, "An overview of load balancing in hetnets: Old myths and open problems," IEEE Wireless Commun., vol. 21, no. 2, pp. 18-25, Apr. 2014.

[19] T. Bu, L. Li, and R. Ramjee, "Generalized proportional fair scheduling in third generation wireless data networks," in Proc. INFOCOM, Apr. 2006, pp. 1-12.

[20] L. Li, M. Pal, and Y. Yang, "Proportional fairness in multi-rate wireless LANs," in Proc. INFOCOM, Apr. 2008, pp. 1-9.

[21] K. Son, S. Chong, and G. Veciana, "Dynamic association for load balancing and interference avoidance in multi-cell networks," IEEE Trans. Wireless Commun., vol. 8, no. 7, pp. 3566-3576, Jul. 2009.

[22] H. Burchardt, S. Sinanovic, Z. Bharucha, and H. Haas, "Distributed and autonomous resource and power allocation for wireless networks," IEEE Trans. Commun., vol. 61, no. 7, pp. 2758-2771, Jul. 2013.

[23] F. Heliot, M. Imran, and R. Tafazolli, "Low-complexity energy-efficient resource allocation for the downlink of cellular systems," IEEE Trans. Commun., vol. 61, no. 6, pp. 2271-2281, Jun. 2013.

[24] M. Ismail and W. Zhuang, "A distributed multi-service resource allocation algorithm in heterogeneous wireless access medium," IEEE J. Sel. Areas Commun., vol. 30, no. 2, pp. 425-432, Feb. 2012.

[25] Q. Ye et al., "User association for load balancing in heterogeneous cellular networks," IEEE Trans. Wireless Commun., vol. 12, no. 6, pp. 2706-2716, Jun. 2013.

[26] F. Jin, R. Zhang, and L. Hanzo, "Resource allocation under delayguarantee constraints for heterogeneous visible-light and RF femtocell," IEEE Trans. Wireless Commun. (Accepted), vol. 14, Feb. 2015.

[27] F. R. Gfeller and U. Bapst, "Wireless in-house data communication via diffuse infrared radiation," Proc. IEEE, vol. 67, no. 11, pp. 1474-1486, Nov. 1979.

[28] H. Zhang and H. Dai, "Cochannel interference mitigation and cooperative processing in downlink multicell multiuser MIMO networks," EURASIP J. Wireless Commun. Netw., vol. 2004, no. 2, pp. 222-235, Dec. 2004.

[29] R. Zhang and L. Hanzo, "Cooperative downlink multicell preprocessing relying on reduced-rate back-haul data exchange," IEEE Trans. Veh. Technol., vol. 60, no. 2, pp. 539-545, Feb. 2011.

[30] F. Kelly, "Charging and rate control for elastic traffic," Eur. Trans. Telecommun., vol. 8, no. 1, pp. 33-37, Jan./Feb. 1997.

[31] J. Akhtman and L. Hanzo, "Power versus bandwidth-efficiency in wireless communications: The economic perspective," in Proc. IEEE VTC, Sep. 2009, pp. 1-5.

[32] S. P. Boyd and L. Vandenberghe, Convex Optimization. Cambridge, U.K.: Cambridge Univ. Press, 2004

[33] B. Bensaou, D. H. K. Tsang, and K. T. Chan, "Credit-based fair queueing (CBFQ): A simple service-scheduling algorithm for packet-switched networks," IEEE/ACM Trans. Netw., vol. 9, no. 5, pp. 591-604, Oct. 2001.

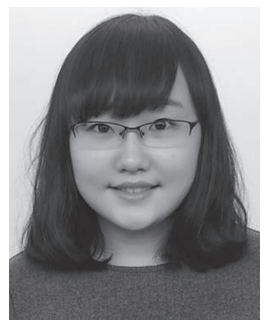

Xuan Li received the B.Eng. degree in optical information science and technology from Beijing Institute of Technology, China, in 2012. She is currently working toward the Ph.D. degree with the Southampton Wireless Group, University of Southampton, UK. Her research interests include visible light communications, heterogeneous networks, resource allocation, and scheduling algorithms.

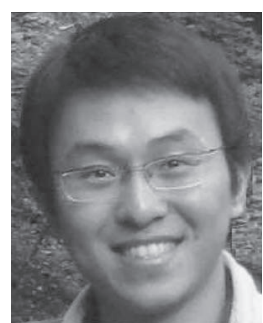

Rong Zhang (M'09) received the B.Sc. degree from Southeast University, China, in 2003, and the Ph.D. degree from Southampton University, UK, in 2009. Before receiving the doctorate, he was an Engineer (August 2003-July 2004) at China Telecom and a Research Assistant (January 2006-May 2009) at Mobile Virtual Center of Excellence (MVCE), U.K. After being a Post-doctoral Researcher (August 2009-July 2012) at Southampton University, he took industrial consulting leave (August 2012-January 2013) for Huawei Sweden R\&D as a System Algorithms Specialist. Since February 13, he has been an appointed as a Lecturer at CSPC group of ECS, Southampton University. He has 40+ journals in prestigious publication avenues (e.g. IEEE, OSA) and many more in major conference proceedings. He regularly serves as reviewer for IEEE transactions and journals and has been a TPC Member/Invited Session Chair of major conferences. He is the recipient of joint funding of MVCE and EPSRC and is also a Visiting Researcher under Worldwide University Network (WUN). More details can be found at http://www.ecs.soton.ac.uk/people/rz

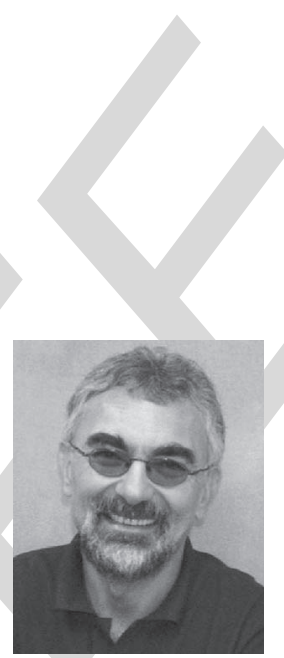

Lajos Hanzo received the bachelor's degree in electronics in 1976 and the doctorate degree in 1983. In 2009 he was awarded the Doctor Honoris Causa by the Technical University of Budapest. During his 38-year career in telecommunications, he has held various research and academic posts in Hungary, Germany, and the U.K. Since 1986 he has been with the School of Electronics and Computer Science, University of Southampton, U.K, where he holds the chair in telecommunications. He has successfully supervised about $100 \mathrm{Ph} . \mathrm{D}$. students, co-authored 20 John Wiley/IEEE Press books on mobile radio communications totalling in excess of 10000 pages, published over 1400 research entries at IEEE Xplore, acted both as TPC and General Chair of IEEE conferences, presented keynote lectures and has been awarded a number of distinctions. Currently, he is directing a 100 -strong academic research team, working on a range of research projects in the field of wireless multimedia communications sponsored by industry, the Engineering and Physical Sciences Research Council (EPSRC) U.K., the European Research Council's Advanced Fellow Grant and the Royal Society's Wolfson Research Merit Award. He is an enthusiastic supporter of industrial and academic liaison and he offers a range of industrial courses. $\mathrm{He}$ is also a Governor of the IEEE Vehicular Technology Society. From 2008 to 2012, he was the Editor-in-Chief of the IEEE Press and a Chaired Professor at Tsinghua University, Beijing, China. His research is funded by the European Research Council's Senior Research Fellow Grant. For further information on his research in progress and associated publications please refer to http:// www-mobile.ecs.soton.ac.uk. 